\title{
THE SYMPLECTIC GEOMETRY OF POLYGONS IN HYPERBOLIC 3-SPACE*
}

\author{
MICHAEL KAPOVICH ${ }^{\dagger}$, JOHN J. MILLSON ${ }^{\ddagger}$, AND THOMAS TRELOAR ${ }^{\S}$
}

\begin{abstract}
We study the symplectic geometry of the moduli spaces $M_{r}=M_{r}\left(\mathbb{H}^{3}\right)$ of closed n-gons with fixed side-lengths in hyperbolic three-space. We prove that these moduli spaces have almost canonical symplectic structures. They are the symplectic quotients of $B^{n}$ by the dressing action of $S U(2)$ (here $B$ is the subgroup of the Borel subgroup of $S L_{2}(\mathbb{C})$ defined below). We show that the hyperbolic Gauss map sets up a real analytic isomorphism between the spaces $M_{r}$ and the weighted quotients of $\left(S^{2}\right)^{n}$ by $P S L_{2}(\mathbb{C})$ studied by Deligne and Mostow. We construct an integrable Hamiltonian system on $M_{r}$ by bending polygons along nonintersecting diagonals. We describe angle variables and the momentum polyhedron for this system. The results of this paper are the analogues for hyperbolic space of the results of [KM2] for $M_{r}\left(\Xi^{3}\right)$, the space of n-gons with fixed side-lengths in $\mathbb{E}^{3}$. We prove $M_{r}\left(\mathbb{H}^{3}\right)$ and $M_{r}\left(\mathbb{E}^{3}\right)$ are symplectomorphic.
\end{abstract}

1. Introduction. An (open) n-gon $P$ in hyperbolic space $\mathbb{H}^{3}$ is an ordered $(\mathrm{n}+1)$-tuple $\left(x_{1}, \ldots, x_{n+1}\right)$ of points in $\mathbb{H}^{3}$ called the vertices. We join the vertex $x_{i}$ to the vertex $x_{i+1}$ by the unique geodesic segment $e_{i}$, called the $i$-th edge. We let $\mathrm{Pol}_{n}$ denote the space of n-gons in $\mathbb{H}^{3}$. An n-gon is said to be closed if $x_{n+1}=x_{1}$. We let $\mathrm{CPol}_{n}$ denote the space of closed n-gons. Two n-gons $P=\left[x_{1}, \ldots, x_{n+1}\right]$ and $P^{\prime}=\left[x_{1}^{\prime}, \ldots, x_{n+1}^{\prime}\right]$ are said to be equivalent if there exists $g \in P S L_{2}(\mathbb{C})$ such that $g x_{i}=x_{i}^{\prime}$, for all $1 \leq i \leq n+1$. We will either represent an n-gon $P$ by its vertices or its edges, $P=\left[x_{1}, \ldots, x_{n+1}\right]=\left(e_{1}, \ldots e_{n}\right)$.

Let $r=\left(r_{1}, \ldots, r_{n}\right)$ be an n-tuple of positive numbers. This paper is concerned with the symplectic geometry of the space of closed n-gons in $\mathbb{H}^{3}$ such that the $i$-th edge $e_{i}$ has side-length $r_{i}, 1 \leq i \leq n$, modulo $P S L_{2}(\mathbb{C})$. We will assume in this paper (with the exception of $\S 3$ ) that $r$ is not on a wall of $D_{n}$ (see $\S 2$ ), hence $M_{r}$ is a real-analytic manifold.

The starting point of this paper is (see $§ 4$ )

THEOREM 1.1. The moduli spaces $M_{r}$ are the symplectic quotients obtained from the dressing action of $S U(2)$ on $B^{n}$.

Here $B=A N$ is the subgroup of the Borel subgroup of $S L_{2}(\mathbb{C}), B=\left\{\left(\begin{array}{cc}\lambda & z \\ 0 & \lambda^{-1}\end{array}\right)\right.$ : $\left.\lambda \in \mathbb{R}_{+}, z \in \mathbb{C}\right\} . B$ is given the Poisson Lie group structure corresponding to the Manin triple $(\mathfrak{s l}(2, \mathbb{C}), \mathfrak{s u}(2), \mathfrak{b})$ with $\langle$,$\rangle on \mathfrak{s l}(2, \mathbb{C})$ given by the imaginary part of the Killing form.

REMARK 1.2. As a consequence of Theorem 1.1, the spaces $M_{r}$ have an almost canonical symplectic structure (the symplectic structure depends on a choice of Iwasawa decomposition of $S L_{2}(\mathbb{C})$ or a ray in $\mathbb{F}^{3}$, but given two such choices, there exist (infinitely many) $g \in S L_{2}(\mathbb{C})$ inducing an isomorphism of the two Poisson structures).

*Received February 3, 2000; accepted for publication April 5, 2000.

†Department of Mathematics, University of Utah, Salt Lake City, UT 84112, USA (kapovich @math.utah.edu). Research partially supported by NSF grant DMS-96-26633.

$\ddagger$ Department of Mathematics, University of Maryland, College Park, MD 20742, USA (jjm@ math.umd.edu). Research partially supported by NSF grant DMS-98-03520.

$\S$ Department of Mathematics, University of Maryland, College Park, MD 20742, USA (txt@ math.umd.edu). 
Our next theorem relates the moduli spaces $M_{r}$ to the weighted quotients $Q_{s s t}=$ $Q_{s s t}(r)$ of $\left(S^{2}\right)^{n}$ constructed by Deligne and Mostow in [DM]. By extending the sides of the n-gon in the positive direction until they meet $S^{2}=\partial_{\infty} \mathbb{H}^{3}$, we obtain a map, the hyperbolic Gauss map $\gamma: \operatorname{Pol}_{n} \rightarrow\left(S^{2}\right)^{n}$. We then have (here we assume $M_{r}$ is smooth)

THEOREM 1.3. The hyperbolic Gauss map induces a real analytic diffeomorphism $\gamma: M_{r} \rightarrow Q_{s s t}(r)$.

REMARK 1.4. In [KM2], the first two authors constructed an analogous analytic isomorphism $\gamma: M_{r}\left(\mathbb{E}^{3}\right) \rightarrow Q_{\text {sst }}(r)$ where $M_{r}\left(\mathbb{E}^{3}\right)$ is the moduli space of $n$-gons with the side-lengths $r=\left(r_{1}, \ldots, r_{n}\right)$ in Euclidean space $\mathbb{E}^{3}$. Although they gave a direct proof, this latter result was a consequence of the Kirwan-Kempf-Ness theorem, [Ki],[KN], relating Mumford quotients to symplectic quotients. Our new result (Theorem 1.3 above) relates a Mumford quotient to a quotient of a symplectic manifold by a Poisson action.

The key step (surjectivity) in the proof of Theorem 1.3 is of independent interest. We could try to invert $\gamma: M_{r} \rightarrow Q_{s s t}$ as follows. Suppose we are given $\xi=\left(\xi_{1}, \ldots, \xi_{n}\right) \in Q_{s s t}$. We wish to construct $P \in M_{r}$ with $\gamma(P)=\xi$. Choose $x \in \mathbb{H}^{3}$. Put the first vertex $x_{1}=x$. Let $\sigma_{1}$ be the geodesic ray from $x_{1}$ to $\xi_{1}$. Let $x_{2}$ be the point on $\sigma_{1}$ with $d\left(x_{1}, x_{2}\right)=r_{1}$. Let $\sigma_{2}$ be the ray from $x_{2}$ to $\xi_{2}$. Cut off $\sigma_{2}$ at $x_{3}$ so that $d\left(x_{2}, x_{3}\right)=r_{2}$. We continue in this way until we get $P=\left[x_{1}, \ldots, x_{n+1}\right]$. However it may not be the case that $P$ closes up (i.e. $x_{n+1}=x_{1}$ ).

THEOREM 1.5. Suppose $\xi$ is a stable configuration (see $\S 3.1)$ on $\left(S^{2}\right)^{n}$. Then there is a unique choice of initial point $x=x(r, \xi)$ such that $P$ closes up.

REMARK 1.6. Let $\widetilde{\nu}$ be the atomic "measure" on $S^{2}$ which assigns mass $r_{i}$ to the point $\xi_{i}, 1 \leq i \leq n$, keeping track of the order of the $\xi_{i}$ 's. Then the rule that assigns $x=x(\widetilde{\nu})=x(\xi, r)$ above is $P S L_{2}(\mathbb{C})$-equivariant and is a multiplicative analogue of the conformal center of mass, $C(\nu)$, of Douady and Earle [DE], see also [MZ, §4]. Here $\nu$ is the measure $\nu=\sum_{i=1}^{n} r_{i} \delta\left(\xi-\xi_{i}\right)$.

REMARK 1.7. We may use Theorem 1.3 to construct a length-shrinking flow on $\mathrm{CPol}_{n}$. Namely, let $0 \leq t \leq 1$. Replace the weights $r=\left(r_{1}, \ldots, r_{n}\right)$ by $t r=$ $\left(t r_{1}, \ldots, t r_{n}\right)$. We have

$$
M_{r} \stackrel{\gamma_{r}}{\longrightarrow} Q_{s s t}(r) \cong Q_{s s t}(t r) \stackrel{\gamma_{t r}}{\longleftarrow} M_{t r} .
$$

The composition $\gamma_{t r}^{-1} \circ \gamma_{r}$ is the length-shrinking flow. Note that $Q_{s s t}(r)$ and $Q_{s s t}(t r)$ are canonically isomorphic as complex analytic spaces. We obtain a curve $x(t)=$ $x(t r, \xi)$. We have

THEOREM 1.8. $\lim _{t \rightarrow 0} x(t r, \xi)=C(\nu)$, the conformal center of mass of Douady and Earle.

REMARK 1.9. We see that $C(\nu)$ is "semi-classical," it depends only on the limit as the curvature goes to zero (or the speed of light goes to infinity), see §3.3.

Our final theorems are connected with the study of certain integrable systems on $M_{r}$ obtained by "bending an n-gon along nonintersecting diagonals" Precisely, we 
proceed as follows. We define the diagonal $d_{i j}$ of $P$ to be the geodesic segment joining $x_{i}$ to $x_{j}$. Here we assume $i<j$. We let $\ell_{i j}$ be the length of $d_{i j}$. Then $\ell_{i j}$ is a continuous function on $M_{r}$ but is not smooth at the points where $\ell_{i j}=0$. We have the following description of the Hamiltonian flow of $\ell_{i j}$ (it is defined provided $\ell_{i j} \neq 0$ ).

THEOREM 1.10. The Hamiltonian flow $\Psi_{i j}^{t}$ of $\ell_{i j}$ applied to an $n$-gon $P \in M_{r}$ is obtained as follows. The diagonal $d_{i j}$ separates $P$ into two halves. Leave one half fixed and rotate the other half at constant speed 1 around $d_{i j}$.

For obvious reasons we call $\Psi_{i j}^{t}$ "bending along $d_{i j}$."

Definition 1.11. We say two diagonals $d_{i j}$ and $d_{a b}$ of $P$ do not intersect if the interiors of $d_{i j}^{*}$ and $d_{a b}^{*}$ do not intersect, where $d_{i j}^{*}$ (resp. $d_{a b}^{*}$ ) is the diagonal of a convex planar n-gon $P^{*}$ corresponding to $d_{i j}$ (resp. $d_{a b}$ ). We then have

THEOREM 1.12. Suppose $d_{i j}$ and $d_{a b}$ do not intersect, then

$$
\left\{\ell_{i j}, \ell_{a b}\right\}=0 \text {. }
$$

REMARK 1.13. We give two proofs of this theorem. The first is a direct computation of the Poisson bracket due to Hermann Flaschka. The second is an elementary geometric one depending on the description of the flows in Theorem 1.10. It corresponds to the geometric intuition that we may wiggle flaps of a folded piece of paper independently if the fold lines do not intersect.

We obtain a maximal collection of commuting flows if we draw a maximal collection of nonintersecting diagonals $\left\{d_{i j},(i, j) \in I\right\}$. Later we will take the collection of all diagonals starting at the first vertex, $I=\{(1,3),(1,4), \ldots,(1, n-1)\}$. Each such collection corresponds to a triangulation of a fixed convex planar n-gon $P^{*}$. There are $n-3$ diagonals in such a maximal collection. Since $\operatorname{dim} M_{r}=2 n-6$, we obtain

THEOREM 1.14. For each triangulation of a convex planar $n$-gon $P^{*}$ we obtain an integrable system on $M_{r}$. Precisely, we obtain a Hamiltonian action of an (n-3)-torus on $M_{r}$ which is defined on the Zariski open subset $M_{r}^{\prime}$ defined by the nonvanishing of the lengths of the diagonals in the triangulation.

We have a simple description of the angle variables and the momentum polyhedron attached to the above integrable system. Let $M_{r}^{o} \subset M_{r}^{\prime}$ be the subset such that none of the $n-2$ triangles in the triangulation are degenerate. Let $\widehat{\theta}_{i j}$ be the dihedral angle at $d_{i j}$. Put $\theta_{i j}=\pi-\widehat{\theta}_{i j}$. Then the $\theta_{i j}$ are angle variables.

To obtain the momentum polyhedron we follow $[\mathrm{HK}]$ and note that there are three triangle inequalities associated to each of the $n-2$ triangles in the triangulation. These are linear inequalities in the $\ell_{i j}$ 's and the $r_{i j}$ 's. If they are satisfied, we can build the $n-2$ triangles then glue them together and get an n-gon $P$ with the required side-lengths $r_{i}$ and diagonal lengths $\ell_{i j}$. We obtain

THEOREM 1.15. The momentum polyhedron of the above torus action (the image of $M_{r}$ under the $\ell_{i j}$ 's) is the subset of $\left(\mathbb{R}_{\geq 0}\right)^{n-3}$ defined by the $3(n-2)$ triangle inequalities above.

As a consequence we obtain 
COROLlaRY 1.16. The functions $\ell_{i j},(i, j) \in I$, are functionally independent.

Our results on $n$-gon linkages in $\mathbb{H}^{3}$ are the analogues of those of [KM2] for $n$ gon linkages in $\mathbb{E}^{3}$. We conclude the paper by comparing the symplectic manifolds $M_{r}\left(\mathbb{H}^{3}\right)$ and $M_{r}\left(\mathbb{E}^{3}\right)$. Assume henceforth that $r$ is not on a wall of $D_{n}$.

Since the Euclidean Gauss map $\gamma_{e}: M_{r}\left(\mathbb{H}^{3}\right) \rightarrow Q_{s s t}(r)$ is a canonical diffeomorphism as is the hyperbolic Gauss map $\gamma_{h}: M_{r}\left(\mathbb{E}^{3}\right) \rightarrow Q_{s s t}(r)$ we obtain

THEOREM 1.17. The hyperbolic and Euclidean Gauss maps induce a canonical diffeomorphism

$$
M_{r}\left(\mathbb{E}^{3}\right) \simeq M_{r}\left(\mathbb{H}^{3}\right)
$$

The last part of the paper is devoted to proving

TheOREM 1.18. $M_{r}\left(\mathbb{E}^{3}\right)$ and $M_{r}\left(\mathbb{H}^{3}\right)$ are (noncanonically) symplectomorphic.

This theorem is proved as follows. Let $X_{\kappa}$ be the complete simply-connected Riemannian manifold of constant curvature $\kappa$. In [Sa], Sargent proved that there exists $\alpha>0$ and an analytically trivial fiber bundle $\pi: \mathcal{E} \rightarrow(-\infty, \alpha)$ such that $\pi^{-1}(\kappa)=M_{r}\left(X_{\kappa}\right)$. We construct a closed relative 2-form $\omega_{\kappa}$ on $\left.\mathcal{E}\right|_{(-\infty, 0]}$ such that $\omega_{\kappa}$ induces a symplectic form on each fiber of $\pi$ and such that the family of cohomology classes $\left[\omega_{\kappa}\right]$ on $\left.\mathcal{E}\right|_{(-\infty, 0]}$ is parallel for the Gauss-Manin connection. Theorem 1.18 then follows from the Moser technique [Mo].

The results are closely related to but different from those of [GW] and [A].

ACKNOWLEDGMENTS. It is a pleasure to thank Hermann Flaschka for his help and encouragement. He explained to us the set-up for the Sklyanin bracket (see §4.1) and provided us with the first proof of Theorem 1.12. Also, this paper was inspired by reading [FR] when we realized that the dressing action of $S U(2)$ on $B^{n}$ was just the natural action of $S U(2)$ on based hyperbolic n-gons. We would also like to thank Jiang-Hua Lu for explaining the formulas of $\S 5.1$ to us. We would also like to thank her for pointing out that it was proved in [GW] that the cohomology class of the symplectic forms $\omega_{\epsilon}$ on an adjoint orbit in the Lie algebra of a compact group was constant.

2. Criteria for the moduli spaces to be smooth and nonempty. In this chapter we will give necessary and sufficient conditions for the moduli space $M_{r}$ to be nonempty and sufficient conditions for $M_{r}$ to be a smooth manifold.

First we need some more notation. Let $*$ be the point in $\mathbb{H}^{3}$ which is fixed by $P S U(2)$. We let $\operatorname{Pol}_{n}(*)$ denote the space of n-gons $\left[x_{1}, \ldots, x_{n+1}\right]$ with $x_{1}=*$ and $\operatorname{CPol}_{n}(*)=\mathrm{CPol}_{n} \cap \operatorname{Pol}_{n}(*)$. We let $\widetilde{N}_{r} \subset \operatorname{Pol}_{n}(*)$ be the subspace of those n-gons $P=\left[x_{1}, \ldots, x_{n+1}\right]$ such that $d\left(x_{i}, x_{i+1}\right)=r_{i}, 1 \leq i \leq n$. We put $N_{r}=\tilde{N}_{r} / P S U(2)$ and $\widetilde{M}_{r}=\widetilde{N}_{r} \cap C \operatorname{Pol}_{n}(*)$. Hence, $M_{r}=\widetilde{M}_{r} / P S U(2)$.

Let $\pi: C P$ l $_{n} \rightarrow\left(\mathbb{R}_{\geq 0}\right)^{n}$ be the map that assigns to an n-gon $e$ its set of sidelengths. $\pi(e)=\left(r_{1}, \ldots, r_{n}\right)$ with $r_{i}=d\left(x_{i}, x_{i+1}\right), 1 \leq i \leq n$.

LemMA 2.1. The image of $\pi$ is the closed polyhedral cone $D_{n}$ defined by the inequalities

$$
r_{1} \geq 0, \ldots, r_{n} \geq 0
$$


and the triangle inequalities

$$
r_{i} \leq r_{1}+\cdots+\hat{r}_{i}+\cdots+r_{n}, 1 \leq i \leq n
$$

(here the "means that $r_{i}$ is omitted).

Proof. The proof is identical to the proof of the corresponding statement for Euclidean space, [KM1, Lemma 1]. $\square$

We next give sufficient conditions for $M_{r}$ to be a smooth manifold. We will use two results and the notation from $\S 4.3$ (the reader will check that no circular reasoning is involved here). By Theorem 4.27 we find that $M_{r}$ is a symplectic quotient.

$$
M_{r} \cong\left(\left.\varphi\right|_{\tilde{N}_{r}}\right)^{-1}(1) / S U(2)
$$

By Lemma 4.23, 1 is a regular value of $\varphi$ unless there exists $P \in \widetilde{M}_{r}$ such that the infinitesimal isotropy $\left.\left(s u_{2}\right)\right|_{P}=\{x \in S U(2): \hat{X}(P)=0\}$ is nonzero.

Definition 2.2. An n-gon $P$ is degenerate if it is contained in a geodesic.

We now have

LEMMA 2.3. $M_{r}$ is singular only if there exists a partition $\{1, \ldots, n\}=I \amalg J$ with $\#(I)>1, \#(J)>1$ such that

$$
\sum_{i \in I} r_{i}=\sum_{j \in J} r_{J}
$$

Proof. Clearly $\left.\left(s u_{2}\right)\right|_{P}=0$ unless $P$ is degenerate. But if $P$ is degenerate there exists a partition $\{1, \ldots, n\}=I \amalg J$ as above ( $I$ corresponds to the back-tracks and $J$ to the forward-tracks of $P$ ).

REMARK 2.4. In the terminology of [KM1], [KM2], $M_{r}$ is smooth unless $r$ is on a wall of $D_{n}$. Note that if $|I|=1$ or $|J|=1$ then $r \in \partial D_{n}$ and $M_{r}$ is reduced to a single point.

There is a technical point concerning smoothness. We could also define $M_{r}$ as the fiber of $\bar{\pi}: C P \mathrm{ol}_{n} / P S L_{2}(\mathbb{C}) \rightarrow D_{n}$ over $r$. It is not quite immediate that smoothness of the symplectic quotient coincides with the smoothness of $\bar{\pi}^{-1}(r)$. Fortunately, this is the case (note $r$ is a regular value of $\bar{\pi} \Leftrightarrow r$ is a regular value of $\pi$ ).

LEMMA 2.5. $r$ is a regular value of $\pi \Leftrightarrow 1$ is a regular value of $\varphi \mid \tilde{N}_{r}$.

Proof. The lemma follows from a consideration of the diagram

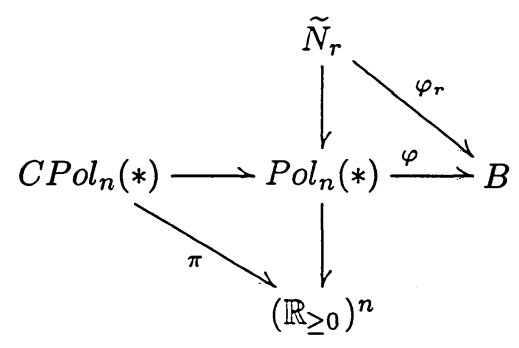


and the observation that $\varphi: \operatorname{Pol}_{n}(*) \rightarrow B$ (see $\left.\S 4.2\right)$ and the side-length map $\operatorname{Pol}_{n}(*) \rightarrow\left(\mathbb{R}_{\geq 0}\right)^{n}$ are obviously submersions. Here we have abbreviated $\varphi \mid \tilde{N}_{r}$ to $\varphi_{r} . \mathrm{Q}$

\section{The geometric invariant theory of hyperbolic polygons.}

3.1. The hyperbolic Gauss map and weighted quotients of the configuration spaces of points on the sphere. The goal of the next two sections is to construct a natural homeomorphism $\gamma: M_{r} \rightarrow Q_{s s t}$ where $Q_{s s t}$ is the $r$-th weighted quotient of $\left(S^{2}\right)^{n}$ by $P S L_{2}(\mathbb{C})$ constructed in [DM] in the case that $M_{r}$ is smooth. $Q_{s s t}$ is a complex analytic space. We now review the construction of $Q_{s s t}$.

Let $M \subset\left(S^{2}\right)^{n}$ be the set of n-tuples of distinct points. Then $Q=M / P S L_{2}(\mathbb{C})$ is a (noncompact) Hausdorff manifold.

Definition 3.1. A point $\vec{u} \in\left(S^{2}\right)^{n}$ is called r-stable (resp. semi-stable) if

$$
\sum_{u_{j}=v} r_{j}<\frac{|r|}{2}\left(\text { resp. } \leq \frac{|r|}{2}\right)
$$

for all $v \in S^{2}$. Here $|r|=\sum_{j=1}^{n} r_{j}$. The set of stable and semi-stable points will be denoted by $M_{\text {st }}$ and $M_{\text {sst }}$ respectively. A semi-stable point $\vec{u} \in\left(S^{2}\right)^{n}$ is said to be a nice semi-stable point if it is either stable or the orbit $P S L_{2}(\mathbb{C}) \vec{u}$ is closed in $M_{\text {sst }}$.

We denote the space of nice semi-stable points by $M_{n s s t}$. We have the inclusions

$$
M_{s t} \subset M_{n s s t} \subset M_{s s t} \text {. }
$$

Let $M_{\text {cusp }}=M_{\text {sst }}-M_{\text {st }}$. We obtain the points $M_{\text {cusp }}$ in the following way. Partition $S=\{1, \ldots, n\}$ into disjoint sets $S=S_{1} \cup S_{2}$ with $S_{1}=\left\{i_{1}, \ldots, i_{k}\right\}, S_{2}=\left\{j_{1}, \ldots, j_{n-k}\right\}$ in such a way that $r_{i_{1}}+\cdots+r_{i_{k}}=\frac{|r|}{2}$ (whence $r_{j_{1}}+\cdots+r_{j_{n-k}}=\frac{|r|}{2}$ ). Then $\vec{u}$ is in $M_{\text {cusp }}$ if either $u_{i_{1}}=\cdots=u_{i_{k}}$ or $u_{j_{1}}=\cdots=u_{j_{n-k}}$. The reader will verify that $\vec{u} \in M_{\text {cusp }}$ is a nice semi-stable point if and only if both sets of the equations above hold. relation $\mathcal{R}$ via:

$\vec{u} \equiv \vec{w}(\bmod \mathcal{R})$ if either

(a) $\vec{u}, \vec{w} \in M_{s t}$ and $\vec{w} \in P S L_{2}(\mathbb{C}) \vec{u}$, or

(b) $\vec{u}, \vec{w} \in M_{\text {cusp }}$ and the partitions of $S$ corresponding to $\vec{u}, \vec{w}$ coincide.

The reader will verify that $\vec{u}, \vec{w} \in M_{n s s t}-M_{s t}$ then $\vec{u} \equiv \vec{w}(\bmod \mathcal{R})$ if and only if $\vec{w} \in P S L_{2}(\mathbb{C}) \vec{u}$.

It is clear that $\mathcal{R}$ is an equivalence relation. Set

$$
Q_{s s t}=M_{s s t} / \mathcal{R}, Q_{n s s t}=M_{n s s t} / \mathcal{R}, Q_{s t}=M_{s t} / \mathcal{R}, Q_{\text {cusp }}=M_{\text {cusp }} / \mathcal{R}
$$

each with the quotient topology. The elements of $Q_{c u s p}$ are uniquely determined by their partitions. Thus $Q_{\text {cusp }}$ is a finite set. It is clear that each equivalence class in $Q_{\text {cusp }}$ contains a unique $P S L_{2}(\mathbb{C})$-orbit of nice semi-stable points whence the inclusion

$$
M_{n s s t} \subset M_{s s t}
$$

induces an isomorphism

$$
Q_{n s s t}=M_{n s s t} / P S L_{2}(\mathbb{C}) \rightarrow Q_{s s t}
$$


In case $r_{1}, \ldots, r_{n}$ are rational then the quotient space $Q_{s s t}$ can be given a structure of a complex projective variety by the techniques of geometric invariant theory applied to certain equivariant projective embeddings of $\left(S^{2}\right)^{n}$, see [DM, §4.6]. This concludes our review of [DM, §4]. We now establish the connection with the moduli space $M_{r}$.

For the rest of this section (with one exception) we will use the ball model of hyperbolic space (so $*=(0,0,0)$ ). We will compactify $\mathbb{H}^{3}$ by enlarging the open three ball to the closed three ball, thus we add $S^{2}=\partial_{\infty} \mathbb{H}^{3}$. Each point of $S^{2}$ corresponds to an equivalence class of geodesic rays in $\mathbb{H}^{3}$. Two rays $\alpha$ and $\beta$ are equivalent if they are asymptotic, i.e. $\lim _{t \rightarrow \infty} \alpha(t)=\lim _{t \rightarrow \infty} \beta(t)$ in the closed three ball. Intrinsically the equivalent rays are characterized by the property that they are within finite Hausdorff distance from each other.

In what follows all geodesic segments, geodesics and geodesic rays will be parameterized by arc-length. We now define the hyperbolic Gauss map $\gamma$ (in various incarnations). Let $\sigma=[x, y], x, y \in \mathbb{H}^{3}$, be the oriented geodesic segment from $x$ to $y$. Let $\tilde{\sigma}(0)$ be the ray, $\tilde{\sigma}:[0, \infty) \rightarrow \mathbb{H}^{3}$ with $\tilde{\sigma}(0)=x$ and $\tilde{\sigma}(\ell)=y$ (here $\ell=\ell(\sigma)$ is the length of the geodesic segment $\sigma$ ). We define the (forward) Gauss map $\gamma$ on oriented segments by

$$
\gamma(\sigma)=\lim _{t \rightarrow \infty} \tilde{\sigma}(t)
$$

We may now define $\gamma: \tilde{N}_{r} \rightarrow\left(S^{2}\right)^{n}$ by

$$
\gamma(e)=\left(\gamma\left(e_{1}\right), \ldots, \gamma\left(e_{n}\right)\right)
$$

One of the main results of this paper is the following theorem - an analogue for Poisson actions of the theorem of Kirwan, Kempf, and Ness, [Ki], [KN].

THEOREM 3.2 .

(i) $\gamma\left(\widetilde{M}_{r}\right) \subseteq M_{n s s t}$.

(ii) If $P$ is nondegenerate, then $\gamma(P) \in M_{\text {st }}$.

(iii) $\gamma$ induces a real analytic homeomorphism $\gamma: M_{r} \rightarrow Q_{s s t}$.

(iv) $M_{r}$ is smooth if and only if $M_{s t}=M_{s s t}$. In this case $Q_{\text {sst }}$ is also smooth and $\gamma: M_{r} \rightarrow Q_{\text {sst }}$ is an analytic diffeomorphism.

Let $\eta \in S^{2}$. We recall the definition of the geodesic flow $\phi_{\eta}^{t}$ associated to $\eta$. (Strictly speaking, this flow is rather the projection to $\mathbb{H}^{3}$ of the restriction of the geodesic flow on $U T\left(\mathbb{H}^{3}\right)$ to the stable submanifold corresponding to $\eta$.) Given $z \in \mathbb{H}^{3}$ there is a unique arc-length parameterized ray $\sigma$ with $\sigma(0)=z, \lim _{t \rightarrow \infty} \sigma(t)=\eta$. By definition,

$$
\phi_{\eta}^{t}(z)=\sigma(t)
$$

We will also need the definition of the Busemann function $b(x, \xi), x \in \mathbb{H}^{3}, \xi \in$ $\partial_{\infty} \mathbb{H}^{3}$. Let $\sigma$ be an arc-length parameterized geodesic ray from $*$ to $\xi$. Then

$$
b(x, \xi)=\lim _{t \rightarrow \infty}(d(x, \sigma(t))-t) .
$$

Note that for $k \in P S U(2)=\operatorname{Stab}(*)$ we have

$$
b(k x, k \xi)=b(x, \xi) .
$$


Also, in the upper half space for $\mathbb{H}^{3}$, we have

$$
b((x, y, z), \infty)=-\log z .
$$

We now prove

LEMMA 3.3. For fixed $\xi,-\nabla b(x, \xi)$ is the infinitesimal generator of the geodesic flow $\phi_{\xi}^{t}$.

Proof. From the first formula above, it suffices to check this statement in the upper half space model (so $*=(0,0,1))$ for $\xi=\infty$. By the second formula

$$
-\nabla b(x, y, z)=z \frac{\partial}{\partial z} \text {. }
$$

We will now prove (i) and (ii) in the statement of Theorem 3.2 above.

LEMMA 3.4.

(i) $\gamma\left(\widetilde{M}_{r}\right) \subseteq M_{n s s t}$.

(ii) $P$ is nondegenerate $\Leftrightarrow \gamma(P) \in M_{\text {st }}$.

Proof. Let $P \in \widetilde{M}_{r}$ be a polygon with the vertices $x_{1}, \ldots, x_{n+1}=x_{n}$, we will use the notation $x_{i}(t), 0 \leq t \leq r_{i}$, for the parameterized edge $e_{i}$ (so that $x_{i}(0)=x_{i}$ ). We test stability of $\gamma(P)$ with respect to a point $\eta \in S^{2}$. Let $b(x):=b(x, \eta)$ be the corresponding Busemann function. Then for any unit vector $v \in T_{x}\left(\mathbb{H}^{3}\right)$

$$
-\nabla b(x) \cdot v \leq 1
$$

with the equality if and only if the geodesic ray $\exp \left(\mathbb{R}_{+} v\right)$ is asymptotic to $\eta$. Similarly,

$$
-\nabla b(x) \cdot v \geq-1
$$

with the equality if and only if the geodesic ray $\exp \left(\mathbb{R}_{-} v\right)$ is asymptotic to $\eta$. Let $I \subset\{1, \ldots, n\}$ be the subset of indices such that $\gamma\left(e_{i}\right)=\eta$. Let $J$ be the complement of $I$ in $\{1, \ldots, n\}$. Put $r_{I}=\sum_{i \in I} r_{i}, r_{J}=\sum_{j \in J} r_{j}$. Since the polygon $P$ is closed, using (3.1) and (3.2) we get:

$$
\begin{aligned}
0=-\left.b\right|_{x_{1}} ^{x_{n+1}}= & \sum_{i=1}^{n-1} \int_{0}^{r_{i}}-x_{i}^{\prime}(t) \cdot \nabla b\left(x_{i}(t)\right) d t \\
& \geq \sum_{i \in I} r_{i}-\sum_{j \in J} r_{j}=r_{I}-r_{J}
\end{aligned}
$$

with the equality if and only if every edge $e_{j}, j \in J$, is contained in the geodesic through $\eta$ and $x_{j}$. Thus $r_{I} \leq r_{J}$, i.e. $\gamma(P)$ is semi-stable. If $\gamma(P)$ is not stable then each edge $e_{i}, 1 \leq i \leq n$, of $P$ is contained in the geodesic through $\eta$ and $x_{i}$, which implies that this geodesic is the same for all $i$. Hence $P$ is degenerate in this case.

In order to prove that $\gamma: M_{r} \rightarrow Q_{n s s t}$ is injective and surjective, we will first need to study a certain dynamical system $f_{r, \xi} \in \operatorname{Diff}\left(\mathbb{H}^{3}\right)$ attached to the configuration of $n$ points $\xi=\left(\xi_{1}, \ldots, \xi_{n}\right)$ on $S^{2}$ weighted by $r=\left(r_{1}, \ldots, r_{n}\right)$. The weights $r$ will usually be fixed and we will drop $r$ in $f_{r, \xi}$. 
3.2. A dynamical system on $\mathbb{H}^{3}$ and the proof that the Gauss map is an isomorphism. Let $\xi=\left(\xi_{1}, \ldots, \xi_{n}\right) \in\left(S^{2}\right)^{n}$. We define a diffeomorphism $f_{\xi}: \mathbb{H}^{3} \rightarrow \mathbb{H}^{3}$ as follows. Assume that $r=\left(r_{1}, \ldots, r_{n}\right) \in D_{n}$ is given. Let $z \in \mathbb{H}^{3}$ be given. Let $\sigma_{1}$ be the ray emanating from $z$ with $\lim _{t \rightarrow \infty} \sigma_{1}(t)=\xi_{1}$. Put $x_{1}=z$ and $x_{2}=\sigma_{1}\left(r_{1}\right)$. Now let $\sigma_{2}$ be the ray emanating from $x_{2}$ with $\lim _{t \rightarrow \infty} \sigma_{2}(t)=\xi_{2}$. Put $x_{3}=\sigma_{2}\left(r_{2}\right)$. We continue in this way until we obtain $x_{n+1}=\sigma_{n}\left(r_{n}\right)$ where $\sigma_{n}$ is the geodesic ray emanating from $x_{n}$ with $\lim _{t \rightarrow \infty} \sigma_{n}(t)=\xi_{n}$. We define $f_{\xi}$ by $f_{\xi}(z)=x_{n+1}$. Note that the polygon $P=\left(x_{1}, \ldots, x_{n+1}\right)$ belongs to $\tilde{N}_{r}$.

We now give another description of $f_{\xi}$ :

$$
f_{\xi}=\dot{\varphi}_{\xi_{n}}^{r_{n}} \circ \cdots \circ \phi_{\xi}^{r_{1}}
$$

where $\phi_{\xi}^{t}$ is the time $t$ geodesic flow towards $\xi$. We may interpret the previous formula for $f_{\xi}$ as a product (or multiplicative) integral [DF]. Partition the interval [0,1] into $n$ equal subintervals, $0=t_{0}<t_{1}<\cdots<t_{n}=1$. Let $\nu$ be the atomic measure on $[0,1]$ given by $\nu(t)=\sum_{i=0}^{n-1} r_{i+1} \delta\left(t-t_{i}\right)$. Let $\lambda:[0,1] \rightarrow S^{2}$ be the map given by $\lambda \mid\left[t_{i}, t_{i+1}\right)=v_{i}, 0 \leq i \leq n-1$. Define $A:[0,1] \rightarrow C^{\infty}\left(\mathbb{H}^{3}, T\left(\mathbb{H}^{3}\right)\right)$ by $A(t)(z)=$ $\nabla b(z, \lambda(t))$. Then in the notation of [DF],

$$
f_{\nu}=\prod_{0}^{1} e^{A(t) d \nu(t)} .
$$

in $\operatorname{Diff}\left(\mathbb{H}^{3}\right)$.

REMARK 3.5. In fact, in $[D F]$ the only integrals considered take values in $G L_{n}(\mathbb{C})$. We have included the above formula to stress the analogy with the conformal center of mass. The above integral is the multiplicative analogue of the gradient of the averaged Busemann function

$$
\nabla b_{\nu}(z)=\int_{S^{2}} \nabla b(z, \eta)\left(\lambda_{*} d \nu\right)(\eta)
$$

used to define the conformal center of mass (see §3.3).

We will first prove

Proposition 3.6. Suppose $\xi$ consists of three or more distinct points. Then $f_{\xi}$ is a strict contraction. Hence, if $\xi$ is stable, $f_{\xi}$ is a strict contraction.

We will need the following lemma

Lemma 3.7. Let $\xi \in S^{2}$ and $\phi_{\xi}^{t}$ be the geodesic flow towards $\xi$. Then, for each $t>0$,

(i) $d\left(\phi_{\xi}^{t}\left(z_{1}\right), \phi_{\xi}^{t}\left(z_{2}\right)\right) \leq d\left(z_{1}, z_{2}\right)$ with equality if and only if $z_{1}$ and $z_{2}$ belong to the same geodesic $\eta$ with end-point $\xi$.

(ii) If $Z \in T_{z}\left(\mathbb{H}^{3}\right)$ is a tangent vector, then

$$
\left\|D \phi_{\xi}^{t}(Z)\right\| \leq\|Z\|
$$

with equality if and only if $Z$ is tangent to the geodesic $\eta$ through $z$ which is asymptotic to $\xi$. 
Proof. We prove (ii) noting that (ii) implies (i). Use the upper half space model for $\mathbb{H}^{3}$ and send $\xi$ to $\infty$. Then, if $Z=(a, b, c)$ is tangent to $\mathbb{H}^{3}$ at $(x, y, z)$, we have

$$
\phi_{\infty}^{t}(x, y, z)=\left(x, y, e^{t} z\right)
$$

and

$$
\left.\left\|D \phi_{\infty}^{t}(a, b, c)\right\|\right|_{\left(x, y, e^{t} z\right)}=\sqrt{e^{-2 t} a^{2}+e^{-2 t} b^{2}+c^{2}}
$$

REMARK 3.8. 1. In case of equality in (i), the points $z_{1}, z_{2}, \phi_{\xi}^{t}\left(z_{1}\right), \phi_{\xi}^{t}\left(z_{2}\right)$ all belong to $\eta$. along $\eta$.

2. The above lemma also follows from the fact that $D \phi_{\xi}^{t}(Z)$ is a stable Jacobi field

We can now prove the proposition. By the previous lemma, $f_{\xi}=\phi_{\xi_{n}}^{r_{n}} \circ \cdots \circ \phi_{\xi_{1}}^{r_{1}}$ does not increase distance. Suppose then that $d\left(f_{\xi}\left(z_{1}\right), f_{\xi}\left(z_{2}\right)\right)=d\left(z_{1}, z_{2}\right)$. Then,

$$
d\left(\phi_{\xi_{1}}^{r_{1}}\left(z_{1}\right), \phi_{\xi_{1}}^{r_{1}}\left(z_{2}\right)\right)=d\left(z_{1}, z_{2}\right) .
$$

Hence, $z_{1}, z_{2}, \phi_{\xi_{1}}^{r_{1}}\left(z_{1}\right), \phi_{\xi_{1}}^{r_{1}}\left(z_{2}\right)$ are all on the geodesic $\eta_{1}$ joining $z_{1}$ to $\xi_{1}$.

Next,

$$
d\left(\phi_{\xi_{2}}^{r_{2}}\left(\phi_{\xi_{1}}^{r_{1}}\left(z_{1}\right)\right), \phi_{\xi_{2}}^{r_{2}}\left(\phi_{\xi_{1}}^{r_{1}}\left(z_{2}\right)\right)\right)=d\left(\phi_{\xi_{1}}^{r_{1}}\left(z_{1}\right), \phi_{\xi_{1}}^{r_{1}}\left(z_{2}\right)\right) .
$$

Hence, $\phi_{\xi_{1}}^{r_{1}}\left(z_{1}\right), \phi_{\xi_{1}}^{r_{1}}\left(z_{2}\right), \phi_{\xi_{2}}^{r_{2}}\left(\phi_{\xi_{1}}^{r_{1}}\left(z_{1}\right)\right), \phi_{\xi_{2}}^{r_{2}}\left(\phi_{\xi_{1}}^{r_{1}}\left(z_{2}\right)\right)$ are all on the same geodesic. This geodesic is necessarily $\eta_{2}$, the geodesic joining $\phi_{\xi_{1}}^{r_{1}}\left(z_{1}\right)$ to $\xi_{2}$, since it contains $\phi_{\xi_{1}}^{r_{1}}\left(z_{1}\right)$ and $\phi_{\xi_{2}}^{r_{2}}\left(\phi_{\xi_{1}}^{r_{1}}\left(z_{1}\right)\right)$. But since $\eta_{2}$ contains $\phi_{\xi_{1}}^{r_{1}}\left(z_{1}\right)$ and $\phi_{\xi_{1}}^{r_{1}}\left(z_{2}\right)$ it also coincides with $\eta_{1}$. Hence, either $\xi_{2}=\xi_{1}$ or $\xi_{1}$ is the opposite end $\check{\xi}_{1}$ of the geodesic $\eta_{1}$. We continue in this way and find that either $\xi_{i}=\xi_{1}$ or $\xi_{i}=\check{\xi}_{1}$, for all $1 \leq i \leq n$.

Our next goal is to prove that $f_{\xi}$ has a fixed-point in $\mathbb{H}^{3}$. Let $H$ be the convex hull of $\left\{\xi_{1}, \ldots, \xi_{n}\right\}$. Let $\beta_{i}$ be the negative of the Busemann function associated to $\xi_{i}$ so $\beta_{i}$ increases along geodesic rays directed toward $\xi_{i}$. Fix a vector $r=\left(r_{1}, \ldots, r_{n}\right)$ and $r$-stable configuration $\xi=\left(\xi_{1}, \ldots, \xi_{n}\right) \in S^{2}$. For $1 \geq h>0$, we shall consider $f_{h r, \xi}: \mathbb{H}^{3} \rightarrow \mathbb{H}^{3}$ where $h r=\left(h r_{1}, \ldots, h r_{n}\right)$. Note that $f_{h r, \xi}(H) \subset H$.

LEMMA 3.9. There exist open horoballs, $\mathcal{O}_{i}, 1 \leq i \leq n$, centered at $\xi_{i}$, which depend only on $r$ and $\xi$, such that for each $1 \leq i \leq n$, if $x \in \mathcal{O}_{i} \cap H$, then

$$
\beta_{i}\left(f_{h r, \xi}(x)\right)<\beta_{i}(x)
$$

(so $f_{h r, \xi}(x)$ is "further away from" $\xi_{i}$ than $x$ ).

Proof. The angle between any two geodesics asymptotic to $\xi_{i}$ is zero, thus by continuity, for each $\epsilon>0$, there exists a horoball $\mathcal{O}_{i}(\epsilon)$ centered at $\xi_{i}$ so that for each $x \in \mathcal{O}_{i}(\epsilon) \cap H$ and for each point $\xi_{j}$ which is different from $\xi_{i}$, the angle between the geodesic ray from $x$ to $\xi_{j}$ and $\nabla b\left(x, \xi_{i}\right)$ is $\leq \epsilon$. Let $I=\left\{\ell \in\{1, \ldots, n\}: \xi_{\ell}=\xi_{i}\right\}, J:=$ $\{1, \ldots, n\}-I$. Recall the stability condition means:

$$
r_{I}:=\sum_{\ell \in I} r_{\ell}<r_{J}:=\sum_{j \in J} r_{j}
$$


thus we can choose $\pi / 2>\epsilon>0$ so that

$$
r_{I}-\cos (\epsilon) r_{J}<0 .
$$

We define $L$ by $\mathcal{O}_{i}(\epsilon)=\left\{\beta_{i}>L\right\}$ for this choice of $\epsilon$, then we define $\mathcal{O}_{i}$ by $\mathcal{O}_{i}:=$ $\left\{\beta_{i}>L+|r|\right\}$. Pick $x \in H \cap \mathcal{O}_{i}$, this point is the initial vertex of the linkage $P$ with vertices

$$
x_{1}=x, x_{2}=\phi_{\xi_{1}}^{h r_{1}}\left(x_{1}\right), \ldots, x_{n+1}=\phi_{\xi_{n}}^{h r_{n}}\left(x_{n}\right)=f_{h r, \xi}(x) .
$$

Note that since the length of $P$ equals $h|r|$, (and $h \leq 1$ ), the whole polygon $P$ is contained in $H \cap \mathcal{O}_{i}(\epsilon)$. We let $x_{j}(t), t \in\left[0, h r_{j}\right]$ be the geodesic segment connecting $x_{j}$ to $x_{j+1}$ (parameterized by the arc-length). Then,

$$
\begin{aligned}
\beta_{i}\left(x_{n+1}\right)-\beta_{i}\left(x_{1}\right) & =\left.\sum_{k=1}^{n} \beta_{i}(x)\right|_{x_{k}} ^{x_{k+1}} \\
& =\sum_{k=1}^{n} \int_{0}^{h r_{k}} \nabla \beta_{i}\left(x_{k}(t)\right) \cdot x_{k}^{\prime}(t) d t \\
& =\sum_{\ell \in I} \int_{0}^{h r_{\ell}} \nabla \beta_{i}\left(x_{\ell}(t)\right) \cdot x_{\ell}^{\prime}(t) d t+\sum_{j \in J} \int_{0}^{h r_{j}} \nabla \beta_{i}\left(x_{j}(t)\right) \cdot x_{j}^{\prime}(t) d t .
\end{aligned}
$$

Recall that $\left\|\nabla \beta_{i}\left(x_{k}(t)\right)\right\|=1,\left\|x_{k}^{\prime}(t)\right\|=1$, if $\ell \in I$ then

$$
\nabla \beta_{i}\left(x_{\ell}(t)\right) \cdot x_{\ell}^{\prime}(t)=1
$$

if $j \in J$ then

$$
\nabla \beta_{i}\left(x_{j}(t)\right) \cdot x_{j}^{\prime}(t) \leq-\cos (\epsilon)
$$

since $x_{j}(t) \in \mathcal{O}_{i}(\epsilon)$ for each $0 \leq t \leq h r_{j}$. Thus,

$$
\beta_{i}\left(x_{n+1}\right)-\beta_{i}\left(x_{1}\right) \leq h \sum_{\ell \in I} r_{\ell}-h \cos (\epsilon) \sum_{j \in J} r_{j}=h\left(r_{I}-\cos (\epsilon) r_{J}\right)<0 .
$$

口

We let $\mathcal{O}_{i}^{\prime}:=\left\{\beta_{i}>L+2|r|\right\}$, then

Proposition 3.10. $f_{h r, \xi}$ has a fixed point in $K:=H-\bigcup_{i=1}^{n} \mathcal{O}_{i}^{\prime}$.

Proof. We claim that if $x \in H-\bigcup_{i=1}^{n} \mathcal{O}_{i}$ then for all $m \geq 0, f_{\xi}^{(m)}(x) \notin \bigcup_{i=1}^{n} \mathcal{O}_{i}^{\prime}$. We first treat the case $m=1$. Since $d\left(x, f_{\xi}(x)\right) \leq|r|$ we see that $x \in H-\bigcup_{i=1}^{n} \mathcal{O}_{i}$ implies $f_{\xi}(x) \notin \bigcup_{i=1}^{n} \mathcal{O}_{i}^{\prime}$. But if there exist an $m-1$ such that $y=f_{\xi}^{(m-1)}(x) \epsilon$ $\bigcup_{i=1}^{n}\left(\mathcal{O}_{i}-\mathcal{O}_{i}^{\prime}\right)$, then $f_{\xi}^{(m)}(x)=f_{\xi}(y) \notin \bigcup_{i=1}^{n} \mathcal{O}_{i}^{\prime}$ by Lemma 3.9 and the claim is proved.

We find that the sequence $\left\{f_{\xi}^{(m)}(x)\right\}$ is relatively compact and contained in $K$. Let $A \subset K$ be the accumulation set for this sequence. This is a compact subset such that $f_{\xi}(A) \subset A$. If $f_{\xi}$ does not have a fixed point in $A$ then the continuous function $\theta(x):=d\left(x, f_{\xi}(x)\right), x \in A$ is bounded away from zero. Let $x_{0} \in A$ be a point where $\theta$ attains its minimum. However (since $f_{\xi}$ is a strict contraction)

$$
\theta\left(f_{\xi}\left(x_{0}\right)\right)=d\left(f_{\xi}\left(x_{0}\right), f_{\xi}^{2}\left(x_{0}\right)\right)<d\left(x_{0}, f\left(x_{0}\right)\right)=\theta\left(x_{0}\right),
$$


contradiction.

We can now prove Theorem 3.2. We first prove that $\gamma: M_{r} \rightarrow Q_{s s t}$ is injective. This easily reduces to proving that if $P, Q \in \widetilde{M}_{r}$ with $\gamma(P)=\gamma(Q)$, then $P=Q$. Let $x_{1}$ be the first vertex of $P, x_{1}^{\prime}$ be the first vertex of $Q$, and $\xi=\gamma(P)=\gamma(Q)$. Since $P$ closes up, we have $f_{\xi}\left(x_{1}\right)=x_{1}$. Since $Q$ closes up, we have $f_{\xi}\left(x_{1}^{\prime}\right)=x_{1}^{\prime}$. But, $f_{\xi}$ is a strict contraction, hence $x_{1}=x_{1}^{\prime}$. It follows immediately that $P=Q$.

We now prove that $\gamma$ is surjective. Let $\xi \in M_{s t}$. There exists $x \in \mathbb{H}^{3}$ with $f_{\xi}(x)=x$. Let $P$ be the n-gon with $\gamma(P)=\xi$ and first vertex $x$. Then $P$ closes up and we have proved that $\gamma$ is onto the stable points. If $\xi$ is nice semi-stable but not stable, then $\xi=\gamma(P)$ for a suitable degenerate n-gon. Hence, $\gamma$ is surjective and Theorem 3.2 is proved.

REMARK 3.11. We have left the proof that the inverse map to $\gamma: M_{r} \rightarrow Q_{s t}$ is smooth (resp. analytic) in the case $M_{r}$ is smooth to the reader. This amounts to checking that the fixed-point of $f_{\xi}$ depends smoothly (resp. analytically) on $\xi$.

3.3. Connection with the conformal center of mass of Douady and Earle. In this section, we prove Theorem 1.8 of the Introduction. We begin by reviewing the definition of the conformal center of mass $C(\nu) \in \mathbb{H}^{3}$, where $\nu$ is a stable measure on $S^{2}=\partial_{\infty} \mathbb{H}^{3}$. Here we are using

Definition 3.12. A measure $\nu$ on $S^{2}$ is stable if

$$
\nu(\{x\})<\frac{|\nu|}{2}, x \in S^{2}
$$

Here, $|\nu|$ is the total mass of $\nu$.

We define the averaged Busemann function, $b_{\nu}: \mathbb{H}^{3} \rightarrow \mathbb{R}$, by

$$
b_{\nu}(x)=\int_{S^{2}} b(x, \xi) d \nu(\xi)
$$

We recall the following proposition ([DE], [MZ, Lemma 4.11]):

Proposition 3.13. Suppose $\nu$ is stable. The $b_{\nu}$ is strictly convex and has a unique critical point (necessarily a minimum).

DEFINITION 3.14. The conformal center of mass $C(\nu)$ is defined to be the above critical point. Thus,

$$
\left.\nabla b_{\nu}\right|_{C(\nu)}=0
$$

The main point is the following,

LEMMA 3.15. The assignment $\nu \rightarrow C(\nu)$ is $P S L_{2}(\mathbb{C})$-equivariant,

$$
C\left(g_{*} \nu\right)=g C(\nu) .
$$

Here $g_{*} \nu$ is the push-forward of $\nu$ by $g \in P S L(2, \mathbb{C})$.

We now return to the set-up of the previous sections. We are given $r=\left(r_{1}, \ldots, r_{n}\right)$ and a stable configuration $\xi=\left(\xi_{1}, \ldots, \xi_{n}\right) \in\left(S^{2}\right)^{n}$. We have the dynamical system $f_{t r, \xi}$ 
of the previous chapter, with fixed-point $x=x(t r, \xi)$. We put $\nu=\sum_{i=1}^{n} r_{i} \delta\left(\xi-\xi_{i}\right)$, where $\delta$ is the Dirac probability measure supported on the origin in $\mathbb{R}^{3}$. We now have,

LEMMA 3.16.

$$
\left.\frac{d}{d t} f_{t r, \xi}\right|_{t=0}=-\nabla b_{\nu}
$$

Proof. We abbreviate $-\nabla b\left(x, \xi_{i}\right)$, the infinitesimal generator of the geodesic flow associated to $\xi_{i}$, to $X_{i}$. Thus we want to prove

$$
\left.\frac{d}{d t} f_{t r, \xi}\right|_{t=0}=\sum_{i=1}^{n} r_{i} X_{i}
$$

But if $\varphi_{t}$ and $\psi_{t}$ are flows with infinitesimal generators $X$ and $Y$ respectively, then

$$
\begin{aligned}
\left.\frac{d}{d t}\right|_{t=0} \varphi \circ \psi(x) & =\left.\frac{\partial^{2}}{\partial t_{1} \partial t_{2}}\right|_{t_{1}=t_{2}=0} \varphi_{t_{1}} \circ \psi_{t_{2}}(x) \\
& =\left.\frac{\partial}{\partial t_{2}}\right|_{t_{2}=0} \varphi_{o} \circ \psi_{t_{2}}(x)+\left.\frac{\partial}{\partial t_{1}}\right|_{t_{1}=0} \varphi_{t_{1}} \circ \psi_{o}(x) \\
& =\left.\frac{\partial}{\partial t_{2}}\right|_{t_{2}=0} \psi_{t_{2}}(x)+\left.\frac{\partial}{\partial t_{1}}\right|_{t_{1}=0} \varphi_{t_{1}}(x) \\
& =Y(x)+X(x) .
\end{aligned}
$$

Recall that

$$
f_{t r, \xi}(x)=\phi_{\xi_{n}}^{t r_{n}} \circ \phi_{\xi_{n-1}}^{t r_{n-1}} \circ \cdots \circ \phi_{\xi_{1}}^{t r_{1}}(x)
$$

Hence,

$$
\left.\frac{d}{d t}\right|_{t=0} f_{t r, \xi}(x)=\sum_{i=1}^{n} X_{i}(x)
$$

We are ready to prove Theorem 1.8 of the Introduction. We abbreviate $-\nabla b_{\nu}$ by $X$.

THEOREM 3.17. Let $x(t r, \xi)$ be the unique fixed point of $f_{t r, \xi}, 0<t \leq 1$. Then

$$
\lim _{t \rightarrow 0} x(t r, \xi)=C(\nu)
$$

Proof. We note that $f_{0 r, \xi}=$ id. Hence, applying Lemma 3.16, the Taylor approximation of $f_{t r, \xi}(x)$ around $t=0$ is

$$
f_{t r, \xi}(x)=x+t X(x)+t^{2} R(x, t)
$$


(where $R(x, t)$ is smooth). Let $\varphi(x, t)=X(x)+t R(x, t)$. By definition, the conformal center of mass $C(\nu)$ is the unique solution of

$$
\varphi(x, 0)=X(x)=0 .
$$

Since $b_{\nu}$ is strictly convex, if $\nu$ is stable, [MZ, Corollary 4.6], $C(\nu)$ is a nondegenerate zero of $X$ and we may apply the implicit function theorem to solve

$$
\varphi(x, t)=0
$$

for $x$ as a function of $t$ near $(C(\nu), 0)$. Thus, there exists $\delta>0$ and smooth curve, $\hat{x}(t)$, defined for $|t|<\delta$, satisfying

(i) $\varphi(\hat{x}(t), t)=0$

(ii) $\hat{x}(0)=C(\nu)$.

But clearly, (i) implies $f_{t r, \xi}(\hat{x}(t))=\hat{x}(t), 0<t<\delta$. Since the fixed-point of $f_{t r, \xi}, 0<$ $t \leq 1$, is unique, we conclude $\hat{x}(t)=x(t r, \xi), 0<t<\delta$. Hence,

$$
\lim _{t \rightarrow 0} x(t r, \xi)=\lim _{t \rightarrow 0} \hat{x}(t)=C(\nu) .
$$

\section{The Symplectic geometry of $M_{r}\left(\mathbb{H}^{3}\right)$.}

4.1. The Poisson-Lie group structure on $B^{n}$. In this section we let $G$ be any (linear) complex simple group, $B=A N$ be the subgroup of the Borel subgroup such that $N$ is its unipotent radical and $A$ is the connected component of the identity in a maximal split torus over $\mathbb{R}$, and $K$ be a maximal compact subgroup. We will construct a Poisson Lie group structure on $G$ which will restrict to a Poisson Lie group structure on $B$. For the basic notions of Poisson Lie group, Poisson action, etc. we refer the reader to [Lu1], [GW], [LW], and [CP].

Let $R_{g}$ and $L_{g}$ be the action of $g$ on $G$ by the right and left multiplication respectively. Let $\mathfrak{g}$ denote the Lie algebra of $G, \mathfrak{k}$ be the Lie algebra of $K$ and $\mathfrak{b}$ be the Lie algebra of $B$. Then $\mathfrak{g}=\mathfrak{b} \oplus \mathfrak{k}$ and $G=B K$. Let $\rho_{\mathfrak{k}}$ (resp. $\rho_{\mathfrak{b}}$ ) be the projection on $\mathfrak{k}$ (resp. on $\mathfrak{b}$ ). We define $\mathcal{R}:=\rho_{\mathfrak{k}}-\rho_{\mathfrak{b}}$ and let $\frac{1}{2}\langle$,$\rangle be the imaginary part of$ the Killing form on $\mathfrak{g}$. In the direct sum splitting $\mathfrak{g}=\mathfrak{k} \oplus \mathfrak{b}$ we see that $\mathfrak{k}$ and $\mathfrak{b}$ are totally-isotropic subspaces dually paired by $\langle$,$\rangle .$

Let $\varphi \in C^{\infty}(G)$. Define $D \varphi: G \rightarrow \mathfrak{g}$ and $D^{\prime} \varphi: G \rightarrow \mathfrak{g}$ by

$$
\begin{aligned}
& \left\langle D^{\prime} \varphi(g), \nu\right\rangle=\left.\frac{d}{d t}\right|_{t=0} \varphi\left(g e^{t \nu}\right) \\
& \langle D \varphi(g), \nu\rangle=\left.\frac{d}{d t}\right|_{t=0} \varphi\left(e^{t \nu} g\right)
\end{aligned}
$$

for $\nu \in \mathfrak{g}$.

We extend $\langle$,$\rangle to a biinvariant element of C^{\infty}\left(G, S^{2} T^{*}(G)\right)$ again denoted $\langle$,$\rangle .$ Now define $\nabla \varphi \in C^{\infty}\left(G, T^{*}(G)\right)$ by

$$
\langle\nabla \varphi(g), x\rangle=d \varphi_{g}(x), x \in T_{g}(G)
$$

We have

$$
D \varphi(g)=d R_{g}^{-1} \nabla \varphi(g)
$$




$$
D^{\prime} \varphi(g)=d L_{g}^{-1} \nabla \varphi(g)=A d_{g^{-1}} D \varphi(g)
$$

The Sklyanin bracket $\{\varphi, \psi\}$ is defined for $\varphi, \psi \in C^{\infty}(G)$ by

$$
\{\varphi, \psi\}(g)=\frac{1}{2}\left(\left\langle\mathcal{R} D^{\prime} \varphi(g), D^{\prime} \psi(g)\right\rangle-\langle\mathcal{R} D \varphi(g), D \psi(g)\rangle\right)
$$

where $\mathcal{R}=\rho_{\mathfrak{k}}-\rho_{\mathfrak{b}}$.

Lemma 4.1. The bracket $\{\varphi, \psi\}$ is a Poisson bracket on $C^{\infty}(G)$.

Proof. See Theorem 1 of [STS].

Let $w \in C^{\infty}\left(G, \Lambda^{2} T(G)\right)$ be the bivector field corresponding to $\{\cdot, \cdot\}$. We now show that $\{\cdot, \cdot\}$ induces a Poisson bracket on $C^{\infty}(B)$.

Lemma 4.2. $w(b)$ is tangent to $B$, i.e. $w(b) \in \Lambda^{2} T_{b}(B) \subset \Lambda^{2} T(G)$ for all $b \in B$.

Proof. It suffices to prove that if $\varphi$ vanishes identically on $B$ then $\{\varphi, \psi\}$ vanishes identically on $B$ for each $\psi$. However if $\varphi$ vanishes identically on $B$ then $\nabla \varphi(b) \in T_{b} B$ for all $b \in B$. Hence $D \varphi(b) \in \mathfrak{b}, D^{\prime} \varphi \in \mathfrak{b}$ for each $b \in B$. This implies that $\mathcal{R} D \varphi=-D \varphi, \mathcal{R} D^{\prime} \varphi=-D^{\prime} \varphi$ and

$$
2\{\varphi, \psi\}(b)=-\left\langle D^{\prime} \varphi(b), D^{\prime} \psi(b)\right\rangle+\langle D \varphi(b), D \psi(b)\rangle
$$

But $D^{\prime} \varphi(b)=A d_{b^{-1}} D \varphi(b), D^{\prime} \psi(b)=A d_{b^{-1}} D \psi(b)$ and $\langle$,$\rangle is A d$-invariant.

For the next corollary note that $T_{b}^{*}(B)$ is a quotient of $T_{b}^{*}(G)$.

Corollary 4.3. Let $\pi$ be the skew-symmetric 2-tensor on $T^{*}(G)$ corresponding to $w$. Pick $b \in B$ and $\alpha, \beta \in T_{b}^{*}(G)$. Then $\pi \mid b$ depends only on the images of $\alpha$ and $\beta$ in $T_{b}^{*}(B)$.

We will continue to use $\pi$ for the skew-symmetric 2-tensor on $T^{*}(B)$ induced by $\pi$ above.

REMARK 4.4. An argument identical to that above proves that $w(k)$ is tangent to $K$. Hence $\{\cdot, \cdot \cdot\}$ induces a Poisson structure on $K$. With the above structures $K$ and $B$ are sub Poisson Lie subgroups of the Poisson Lie group $G$.

We will need a formula for the Poisson tensor $\pi$ on $B$. We will use $\langle$,$\rangle to identify$ $T^{*}(G)$ and $T(G)$. Under this identification $T_{b}^{*}(B)$ is identified to $T_{b}(G) / T_{b}(B)$. We will identify this quotient with $d R_{b}$ k. We let $\left.\tilde{\pi}\right|_{b}$ denote the resulting skew-symmetric 2-tensor on $d R_{b} \mathfrak{k}$. Finally we define $\tilde{\pi}^{r} \in C^{\infty}\left(B,\left(\Lambda^{2} \mathfrak{k}\right)^{*}\right)$ by

$$
\left.\check{\pi}\right|_{b} ^{r}(x, y)=\left.\check{\pi}\right|_{b}\left(d R_{b} x, d R_{b} y\right), \quad x, y \in \mathfrak{k}
$$

We now recover formulae (2.25) of [FR] (or [LR, Definition 4.2]) for $\check{\pi}^{r}$.

LEMMA 4.5. $\left.\check{\pi}\right|_{b} ^{r}(x, y)=\left\langle\rho_{\mathfrak{k}}\left(A d_{b^{-1}} x\right), \rho_{\mathfrak{b}}\left(A d_{b^{-1}} y\right)\right\rangle$.

Proof. Choose $\varphi, \psi \in C^{\infty}(G)$ with $\nabla \varphi(b)=d R_{b} x$ and $\nabla \psi(b)=d R_{b} y$. Then

$$
\begin{aligned}
\left.\check{\pi}\right|_{b} ^{r}(x, y) & =\left.\check{\pi}\right|_{b}\left(d R_{b} x, d R_{b} y\right)=\left.\check{\pi}\right|_{b}(\nabla \varphi(b), \nabla \psi(b)) \\
& =\left.\pi\right|_{b}(d \varphi(b), d \psi(b))=\{\varphi, \psi\}(b) \\
& =\frac{1}{2}\left\langle\mathcal{R} d L_{b}^{-1} \nabla \varphi(b), d L_{b}^{-1} \nabla \psi(b)\right\rangle-\frac{1}{2}\left\langle\mathcal{R} d R_{b}^{-1} \nabla \varphi(b), d R_{b}^{-1} \nabla \psi(b)\right\rangle \\
& =\frac{1}{2}\left\langle\mathcal{R} A d_{b^{-1}} x, A d_{b^{-1}} y\right\rangle-\frac{1}{2}\langle\mathcal{R} x, y\rangle .
\end{aligned}
$$


But $x, y \in \mathfrak{k}$ implies $\langle\mathcal{R} x, y\rangle=0$. Hence,

$$
\begin{aligned}
\left.\check{\pi}\right|_{b} ^{r}(x, y) & =\frac{1}{2}\left\langle\rho_{\mathfrak{k}}\left(A d_{b^{-1}} x\right), A d_{b^{-1}} y\right\rangle-\frac{1}{2}\left\langle\rho_{\mathfrak{b}}\left(A d_{b^{-1}} x\right), A d_{b^{-1}} y\right\rangle \\
& =\frac{1}{2}\left\langle\rho_{\mathfrak{k}}\left(A d_{b^{-1}} x\right), \rho_{\mathfrak{b}} A d_{b^{-1}} y\right\rangle-\frac{1}{2}\left\langle\rho_{\mathfrak{b}}\left(A d_{b^{-1}} x\right), \rho_{\mathfrak{k}} A d_{b^{-1}} y\right\rangle \\
& =\left\langle\rho_{\mathfrak{k}}\left(A d_{b^{-1}} x\right), \rho_{\mathfrak{b}}\left(A d_{b^{-1}} y\right)\right\rangle .
\end{aligned}
$$

The last equality holds by skew-symmetry, see [LR, Lemma 4.3].

We will abuse notation and drop the ${ }^{-}$and $r$ in the notation for $\check{\pi}^{r}$ henceforth.

REMARK 4.6. The Poisson tensor on $K, \pi_{K}$, induced from the Skylanin bracket on $G$ is the negative of the usual Poisson tensor on $K$ (see [FR], [Lu1]). Throughout this paper we let $\pi_{K}(k)=d L_{k} X \wedge Y-d R_{k} X \wedge Y$, where $X=\frac{1}{2}\left(\begin{array}{cc}0 & 1 \\ -1 & 0\end{array}\right)$ and $Y=$ $\frac{1}{2}\left(\begin{array}{cc}0 & i \\ i & 0\end{array}\right)$.

We now give $G^{n}$ the product Poisson structure, hence $B^{n}$ inherits the product structure. We introduce more notation to deal with the product. We let $\mathfrak{g}_{i} \subset \mathfrak{g}^{n}=$ $\mathfrak{g} \oplus \ldots \oplus \mathfrak{g}$ be the image of $\mathfrak{g}$ under the embedding into $i$-th summand. For $\varphi \in C^{\infty}\left(G^{n}\right)$ we define

$$
D_{i} \varphi: G^{n} \rightarrow \mathfrak{g}_{i}, \quad D_{i}^{\prime} \varphi: G^{n} \rightarrow \mathfrak{g}_{i}
$$

as follows. Let $g=\left(g_{1}, \ldots, g_{n}\right) \in G^{n}$ and $\nu \in \mathfrak{g}_{i}$, then

$$
\begin{aligned}
& \left\langle D_{i} \varphi, \nu\right\rangle=\left.\frac{d}{d t}\right|_{t=0} \varphi\left(g_{1}, \ldots, e^{t \nu} g_{i}, \ldots, g_{n}\right) \\
& \left\langle D_{i}^{\prime} \varphi, \nu\right\rangle=\left.\frac{d}{d t}\right|_{t=0} \varphi\left(g_{1}, \ldots, g_{i} e^{t \nu}, \ldots, g_{n}\right)
\end{aligned}
$$

Here we extend $\langle$,$\rangle to \mathfrak{g}^{n}$ by

$$
\langle\delta, \gamma\rangle=\sum_{i=1}^{n}\left\langle\delta_{i}, \gamma_{i}\right\rangle
$$

for $\delta=\left(\delta_{1}, \ldots, \delta_{n}\right), \gamma=\left(\gamma_{1}, \ldots, \gamma_{n}\right)$. We define $d_{i}, \nabla_{i}$ in an analogous fashion. Finally define the Poisson bracket on $C^{\infty}\left(G^{n}\right)$ by

$$
\{\varphi, \psi\}(g)=\frac{1}{2} \sum_{i=1}^{n}\left[\left\langle\mathcal{R} D_{i}^{\prime} \varphi(g), D_{i}^{\prime} \psi(g)\right\rangle-\left\langle\mathcal{R} D_{i} \varphi(g), D_{i} \psi(g)\right\rangle\right] .
$$

As expected we obtain an induced Poisson bracket on $C^{\infty}\left(B^{n}\right)$ using the above formula with $g \in G$ replaced by $b \in B$.

Now let $\pi$ be the Poisson tensor on $G^{n}$ corresponding to the above Poisson bracket. Let $\pi^{\#} \in \operatorname{Hom}\left(T^{*}\left(G^{n}\right), T\left(G^{n}\right)\right)$ be defined by $\beta\left(\pi^{\#}(\alpha)\right)=\pi(\alpha, \beta)$. Let $\varphi \in C^{\infty}\left(G^{n}\right)$. We have

DEFINITION 4.7. The Hamiltonian vector field associated to $\varphi$ is the vector field $X_{\varphi} \in C^{\infty}\left(G^{n}, T\left(G^{n}\right)\right)$ given by

$$
X_{\varphi}=\pi^{\#} d \varphi
$$


We will need a formula for $X_{\varphi}$.

LemMA 4.8. Let $g=\left(g_{1}, \ldots, g_{n}\right)$. Then $X_{\varphi}(g)=\left(X_{1}(g), \ldots, X_{n}(g)\right)$ where

$$
X_{i}(g)=\frac{1}{2}\left[d R_{g_{i}} \mathcal{R} D_{i} \varphi(g)-d L_{g_{i}} \mathcal{R} D_{i}^{\prime} \varphi(g)\right]
$$

Proof. We will use the formula

$$
\{\varphi, \psi\}=-d \psi\left(X_{\varphi}\right)=-\left\langle X_{\varphi}, \nabla \psi\right\rangle
$$

Here $\nabla$ is the gradient with respect to $\langle$,$\rangle on \mathfrak{g}^{n}$, see above, hence $\nabla \psi=\left(\nabla_{1} \psi, \ldots, \nabla_{n} \psi\right)$. We have

$$
\begin{aligned}
\{\varphi, \psi\}(g) & =\frac{1}{2} \sum_{i=1}^{n}\left[\left\langle\mathcal{R} D_{i}^{\prime} \varphi(g), D_{i}^{\prime} \psi(g)\right\rangle-\left\langle\mathcal{R} D_{i} \varphi(g), D_{i} \psi(g)\right\rangle\right] \\
& =\frac{1}{2} \sum_{i=1}^{n}\left[\left\langle\mathcal{R} D_{i}^{\prime} \varphi(g), d L_{g_{i}}^{-1} \nabla_{i} \psi(g)\right\rangle-\left\langle\mathcal{R} D_{i} \varphi(g), d R_{g_{i}}^{-1} \nabla_{i} \psi(g)\right\rangle\right] \\
& =\frac{1}{2} \sum_{i=1}^{n}\left[\left\langle d L_{g_{i}} \mathcal{R} D_{i}^{\prime} \varphi(g), \nabla_{i} \psi(g)\right\rangle-\left\langle d R_{g_{i}} \mathcal{R} D_{i} \varphi(g), \nabla_{i} \psi(g)\right\rangle\right] \\
& =\frac{1}{2}\left\langle\left(X_{1}(g), \ldots, X_{n}(g)\right),\left(\nabla_{1} \psi(g), \ldots, \nabla_{n} \psi(g)\right)\right\rangle \\
& =\frac{1}{2}\left\langle X_{\varphi}(g), \nabla \psi(g)\right\rangle
\end{aligned}
$$

口 $B^{n}$.

REMARK 4.9. Since $w(b)$ is tangent to $B^{n}$ the field $X_{\varphi}(b)$ will also be tangent to

4.2. The dressing action of $K$ on $B^{n}$ and the action on $n$-gons in $G / K$. The basic reference for this section is [FR]. In that paper the authors take $n=2$ and write $G=K B$. We will leave to the reader the task of comparing our formulae with theirs.

In what follows we let $G=S L_{2}(\mathbb{C}), K=S U(2)$, and $B$ be the subgroup of $G$ consisting of upper-triangular matrices with positive diagonal entries. We let $\rho_{B}, \rho_{K}$ be the projections relative to the decomposition $G=B K$. For the next theorem (in the case $n=2$ ) see [FR, Formula 2.15].

Theorem 4.10. There is a Poisson action of $K$ on the Poisson manifold $B^{n}$ given by

$$
k \cdot\left(b_{1}, \ldots, b_{n}\right)=\left(b_{1}^{\prime}, \ldots, b_{n}^{\prime}\right)
$$

with $b_{i}^{\prime}=\rho_{B}\left(\rho_{K}\left(k b_{1} \cdots b_{i-1}\right) b_{i}\right), 1 \leq i \leq n$. 
DEFINITION 4.11. The above action is called the dressing action of $K$ on $B^{n}$.

Definition 4.12. For $n=1$, we denote by $B_{r}=K b$ the dressing orbit of $b$, where $b \in B$ and $d(b *, *)=r$. We will also need the formula for the infinitesimal dressing action of $\mathfrak{k}$ on $B^{n}$. This action is given for $x \in \mathfrak{k}$ by

$$
x \cdot\left(b_{1}, \cdots, b_{n}\right)=\left(\xi_{1}, \ldots, \xi_{n}\right) \in T_{b}\left(B^{n}\right)
$$

with $\xi_{i}=d L_{b_{i}} \rho_{\mathfrak{b}} A d_{b_{i}^{-1}} \rho_{\mathfrak{k}} A d_{\left(b_{1} \cdots b_{i-1}\right)^{-1}} x$. Note that $\xi_{i} \in T_{b_{i}}(B)$.

REMARK 4.13. In order to pass from the $K$-action to the $\mathfrak{k}$-action observe that $\rho_{K}(b g)=\rho_{K}(g)$ and $\rho_{B}(b g)=b \rho_{B}(g)$. Accordingly we may rewrite the $K$-action as $k \cdot\left(b_{1}, \ldots, b_{n}\right)=\left(b_{1}^{\prime}, \ldots, b_{n}^{\prime}\right)$ with

$$
b_{i}^{\prime}=b_{i} \rho_{B}\left(b_{i}^{-1} \rho_{K}\left(\left(b_{1} \cdots b_{i-1}\right)^{-1} k b_{1} \cdots b_{i-1}\right) b_{i}\right), 1 \leq i \leq n .
$$

Recall, $* \in \mathbb{H}^{3}$ is the element fixed by the action of $K, K \cdot *=*$. Since $B$ acts simply-transitively on $G / K$ we have

Lemma 4.14. (i) The map $\Phi: B^{n} \rightarrow \operatorname{Pol}_{n}(*)$ given by

$$
\Phi\left(b_{1}, \ldots, b_{n}\right)=\left(*, b_{1} *, \ldots, b_{1} \cdots b_{n} *\right)
$$

is a diffeomorphism.

(ii) The map $\Phi$ induces a diffeomorphism from $\left\{b \in B^{n}: b_{1} \cdots b_{n}=1\right\}$ onto $\mathrm{CPol}_{n}(*)$.

We now have

Lemma 4.15. $\Phi$ is a $K$-equivariant diffeomorphism where $K$ acts on $B^{n}$ by the dressing action and on $\operatorname{Pol}_{n}(*)$ by the natural (diagonal) action.

Proof. Let $k \cdot\left(b_{1}, \ldots, b_{n}\right)=\left(b_{1}^{\prime \prime}, \ldots, b_{n}^{\prime \prime}\right)$ be the pull-back to $B^{n}$ of the action of $K$ on $\operatorname{Pol}_{n}(*)$. Then

$$
\begin{gathered}
b_{1}^{\prime \prime} *=k b_{1} * \\
b_{1}^{\prime \prime} b_{2}^{\prime \prime} *=k b_{1} b_{2} * \\
\vdots \\
b_{1}^{\prime \prime} \cdots b_{n}^{\prime \prime} *=k b_{1} \cdots b_{n} *
\end{gathered}
$$

We obtain

$$
\begin{gathered}
b_{1}^{\prime \prime} \cdots b_{i}^{\prime \prime}=\rho_{B}\left(k b_{1} \cdots b_{i}\right) \\
b_{i}^{\prime \prime}=\rho_{B}\left(\left(b_{1}^{\prime \prime} \cdots b_{i-1}^{\prime \prime}\right)^{-1} k\left(b_{1} \cdots b_{i}\right)\right)=\rho_{B}\left(\left(\rho_{B}\left(b_{1}^{\prime \prime} \cdots b_{i-1}^{\prime \prime}\right)\right)^{-1} k b_{1} \cdots b_{i-1} b_{i}\right)
\end{gathered}
$$




$$
=\rho_{B}\left(\left(\rho_{B}\left(k b_{1} \cdots b_{i-1}\right)\right)^{-1} k b_{1} \cdots b_{i-1} b_{i}\right)=\rho_{B}\left(\rho_{K}\left(k b_{1} \cdots b_{i-1}\right) b_{i}\right)
$$

There is another formula for the dressing action of $K$ on $B^{n}$ that will be useful.

LEMMA 4.16. With the above notation

$$
\left.\left.b_{i}^{\prime}=\rho_{B}(\underbrace{\rho_{K}\left(\cdots \left(\rho_{K}\right.\right.}_{i-1 \text { times }}\left(k b_{1}\right) b_{2}) \cdots b_{i-1}\right) b_{i}\right)
$$

Proof. Induction on $i$.

We obtain a corresponding formula for the infinitesimal dressing action of $\mathfrak{k}$ on $B^{n}$.

LEMMA 4.17. $x \cdot\left(b_{1}, \ldots, b_{n}\right)=\left(\xi_{1}, \ldots, \xi_{n}\right)$ where

$$
\xi_{i}=d L_{b_{i}} \rho_{\mathfrak{b}} A d_{b_{i}^{-1}} \rho_{\mathrm{E}} A d_{b_{i-1}^{-1}} \cdots \rho_{\mathfrak{k}} A d_{b_{1}^{-1}} x .
$$

We now draw an important consequence.

LEMMA 4.18. The map $\Phi$ induces a diffeomorphism between $B_{r}^{n}=B_{r_{1}} \times \cdots \times B_{r_{n}}$ and the configuration space of open based n-gon linkages $\tilde{N}_{r}$, where if $b \in B_{r}^{n}$, then $r=\left(r_{1}, . ., r_{n}\right)$ and $d\left(b_{1} \cdots b_{i} *, b_{1} \cdots b_{i-1} *\right)=r_{i}$, for all $1 \leq i \leq n$. $\Phi(b)$ is

Proof. Let $b \in B_{r}^{n}$ be given. Then $\Phi(b)=\left(*, b_{1} *, \ldots, b_{1} \cdots b_{n} *\right)$. The $K$-orbit of

$$
\left[*, k b_{1} *, \ldots, k\left(b_{1} \cdots b_{n}\right) *\right]
$$

The $i$-th edge $e_{i}$ of $\Phi(b)$ is the geodesic segment joining $k b_{1} \cdots b_{i-1} *$ to $k b_{1} \cdots b_{i} *$. Clearly this is congruent (by $k b_{1} \cdots b_{i-1}$ ) to the segment connecting $*$ to $b_{i} *$.

CoROllary 4.19. The symplectic leaves of $B^{n}$ map to the configuration spaces $\tilde{N}_{r}$ under $\Phi$.

Proof. The $B_{r}^{n}$ are the symplectic leaves of $B^{n}$.

4.3. The moduli space $M_{r}$ as a symplectic quotient. We have seen that $\Phi$ induces a diffeomorphism from $\left\{\left(b_{1}, \ldots, b_{n}\right) \in B_{r}^{n}: b_{1} \cdots b_{n}=1\right\} / K$ to the moduli space $M_{r}$ of closed $n$-gon linkages in $\mathbb{H}^{3}$ modulo isometry. In this section we will prove that the map $\varphi: B^{n} \rightarrow B$ given by $\varphi\left(b_{1}, \cdots, b_{n}\right)=b_{1} \cdots b_{n}$ is a momentum map for the (dressing) $K$ action on $B^{n}$. Hence, $M_{r}$ is a symplectic quotient, in particular it is a symplectic manifold if 1 is a regular value by Lemma 4.24 below.

The definition of a momentum map for a Poisson action of a Poisson Lie group was given in [Lu1].

Definition 4.20. Suppose that $K$ is a Poisson Lie group, $(M, \pi)$ is a Poisson manifold, and $K \times M \rightarrow M$ is a Poisson action. Let $x \in \mathfrak{E}, \alpha_{x}$ be the extension of 
$x \in \mathfrak{k}=\left(\mathfrak{k}^{*}\right)^{*}$ to a right-invariant 1-form on $K^{*}$, and $\hat{x}$ be the induced vector field on $M$. Then a map $\varphi: M \rightarrow K^{*}$ is a momentum map if it satisfies the equation

$$
-\pi^{\#} \varphi^{*} \alpha_{x}=\hat{x}
$$

Remark 4.21. For the definition of $K^{*}$, the dual Poisson Lie group, see [Lu1]. In our case $K^{*}=B$.

The next lemma is proved in [Lu1] and [Lu3]. We include a proof here for completeness.

Lemma 4.22. Suppose that $M$ is a symplectic manifold, $K$ is a Poisson Lie group and $K \times M \rightarrow M$ is a Poisson action with an equivariant momentum map $\varphi: M \rightarrow K^{*}$. Assume 1 is a regular value of $\varphi$. Then $\varphi^{-1}(1) / K$ is a symplectic orbifold with the symplectic structure given by taking restriction and quotient of the symplectic structure on $M$. If we assume further that the isotropy subgroups of all $x \in \varphi^{-1}(1)$ are trivial then $\varphi^{-1}(1) / K$ is a manifold.

Proof. Let $\omega$ be the symplectic form on $M$ and $m \in \varphi^{-1}(1) \subset M$. Let $V_{m}$ be a complement to $T_{m} \varphi^{-1}(1)$ in $T_{m} M$. Let $\mathfrak{k} \cdot m \subset T_{m} M$ be the tangent space to the orbit $K \cdot m$. Hence $\mathfrak{k}_{m}=\{\hat{x}(m): x \in \mathfrak{k}\}$. We first prove the identity

$$
\omega_{m} \hat{x}(m)=-\left.\varphi^{*} \alpha_{x}\right|_{m}
$$

Here we use $\omega_{m}$ to denote the induced map $T_{m} M \rightarrow T_{m}^{*} M$ as well as the symplectic form evaluated at $m$. Indeed we have the identity

$$
-\pi^{\#} \varphi^{*} \alpha_{x}=\hat{x}
$$

Applying $\omega$ we get

$$
\omega \hat{x}=-\varphi^{*} \alpha_{x}
$$

We claim that if $m \in \varphi^{-1}(1)$ the $\mathfrak{k} \cdot m$ is orthogonal (under $\omega_{m}$ ) to $T_{m}\left(\varphi^{-1}(1)\right.$ ), in particular it is totally-isotropic. Let $\hat{x}(m) \in \mathfrak{k} \cdot m$ and $u \in T_{m}\left(\varphi^{-1}(1)\right)$. Then

$$
\omega_{m}(\hat{x}(m), u)=-\left.\varphi^{*} \alpha_{x}\right|_{m}(u)=-\left.\alpha_{x}\right|_{m}\left(d \varphi_{m} u\right)
$$

But $d \varphi_{m} u=0$ and the claim is proved. Hence, the restriction of $\omega_{m}$ to $T_{m}\left(\varphi^{-1}(1)\right)$ descends to $T_{m}\left(\varphi^{-1}(1)\right) / \mathfrak{k} \cdot m=T_{K \cdot m}\left(\varphi^{-1}(1) / K\right)$. We now prove that the induced form is nondegenerate.

To this end we claim that $\mathfrak{k} \cdot m$ and $V_{m}$ are dually paired by $\omega_{m}$. We draw two conclusions from the hypothesis that 1 is a regular value for $\varphi$. First by [FR, Lemma 4.2] the map $\mathfrak{k} \rightarrow \mathfrak{k} \cdot m$ given by $x \mapsto \hat{x}(m)$ is an isomorphism. Second, $d \varphi_{m}: V_{m} \rightarrow \mathfrak{k}^{*}$ is an isomorphism. Let $\left\{x_{1}, \ldots, x_{N}\right\}$ be a basis for $\mathfrak{k}$, whence $\left\{\hat{x}_{1}(m), \ldots, \hat{x}_{N}(m)\right\}$ is a basis for $\mathfrak{k} \cdot m$. We want to find a basis $\left\{v_{1}, \ldots, v_{N}\right\}$ for $V_{m}$ so that $\omega_{m}\left(\hat{x}_{i}(m), v_{j}\right)=\delta_{i j}$. Choose a basis $\left\{v_{1}, \ldots, v_{N}\right\}$ for $V_{m}$ such that $\left\{d \varphi_{m} v_{1}, \ldots, d \varphi_{m} v_{N}\right\} \subset \mathfrak{k}^{*}$ is dual to $\left\{x_{1}, \ldots, x_{N}\right\}$. Then

$$
\omega_{m}\left(\hat{x}_{i}(m), v_{j}\right)=-\left.\varphi^{*} \alpha_{x_{i}}\right|_{m}\left(v_{j}\right)=-\left.\alpha_{x_{i}}\right|_{m}\left(d \varphi v_{j}\right)=-d \varphi v_{j}\left(x_{i}\right)=-\delta_{i j}
$$


As a consequence of the previous claim, the restriction of $\omega_{m}$ to $\mathfrak{k} \cdot m \oplus V_{m}$ is nondegenerate. We then have the orthogonal complement $\left(\mathfrak{k} \cdot m \oplus V_{m}\right)^{\perp}$ is a complement to $\mathfrak{k} \cdot m \oplus V_{m}$ and $\omega_{m} \mid\left(\mathfrak{k} \cdot m \oplus V_{m}\right)^{\perp}$ is nondegenerate. But then $\left(\mathfrak{k} \cdot m \oplus V_{m}\right)^{\perp}$ maps isomorphically to $T_{K \cdot m}\left(\varphi^{-1}(1) / K\right)$.

We will also need

LEMMA 4.23. $\varphi(m)$ is a regular value for $\varphi$ if and only if $\mathfrak{k}_{m}=\{x \in \mathfrak{k}: \hat{x}(m)=$ $0\}=0$.

Proof. Let $x \in \mathfrak{k}$. Then $x \in\left(\left.\operatorname{Im} d \varphi\right|_{m}\right)^{\perp} \Leftrightarrow \varphi^{*} \alpha_{x}=0 \Leftrightarrow 0=-\pi \varphi^{*} \alpha_{x}=\hat{x}(m)$.

We now begin the proof that $\varphi$ is a momentum map for the dressing action of $K$ on $B^{n}$. We will need some notation. Let $x \in \mathfrak{k}=\mathfrak{b}^{*}$. Recall that $\alpha_{x}$ is the extension of $x$ to a right-invariant 1-form on $B$. Thus if $\zeta \in T_{b}(B)$ we have

$$
\left.\alpha_{x}\right|_{b}(\zeta)=\left\langle d R_{b} x, \zeta\right\rangle
$$

LeMmA 4.24. $-\left.\pi^{\#} \alpha_{x}\right|_{b}=d L_{b} \rho_{b} A d_{b^{-1}} x$.

Proof. Let $y \in \mathfrak{k}$. It suffices to prove that

$$
\left.\pi\right|_{b}\left(\alpha_{x}(b), \alpha_{y}(b)\right)=-\left.\alpha_{y}\right|_{b}\left(d L_{b} \rho_{b} A d_{b^{-1}} x\right)
$$

Now

$$
\left.\pi\right|_{b}\left(\alpha_{x}(b), \alpha_{y}(b)\right)=\left.\pi\right|_{b}\left(d R_{b} x, d R_{b} y\right)=\left.\pi^{r}\right|_{b}(x, y)=\left\langle\rho_{\mathfrak{k}}\left(A d_{b^{-1}} x\right), \rho_{\mathfrak{b}}\left(A d_{b^{-1}} y\right)\right\rangle
$$

according to Lemma 4.5. Also

$$
\begin{gathered}
-\left.\alpha_{y}\right|_{b}\left(d L_{b} \rho_{\mathfrak{b}} A d_{b^{-1}} x\right)=-\left\langle d R_{b} y, d L_{b} \rho_{\mathfrak{b}} A d_{b^{-1}} x\right\rangle \\
=-\left\langle A d_{b^{-1}} y, \rho_{\mathfrak{b}} A d_{b^{-1}} x\right\rangle=-\left\langle\rho_{\mathfrak{k}} A d_{b^{-1}} y, \rho_{\mathfrak{b}} A d_{b^{-1}} x\right\rangle=\left\langle\rho_{\mathfrak{k}} A d_{b^{-1}} x, \rho_{\mathfrak{b}} A d_{b^{-1}} y\right\rangle
\end{gathered}
$$

0

LEMMA 4.25. $\left.\quad \varphi^{*} \alpha_{x}\right|_{b}=\left(\left.\alpha_{x_{1}}\right|_{b_{1}}, \cdots,\left.\alpha_{x_{n}}\right|_{b_{n}}\right)$, where $x_{1}=x$ and $x_{i}=$ $\rho_{\mathfrak{k}}\left(A d_{\left(b_{1} \cdots b_{i-1}\right)^{-1}} x\right), 2 \leq i \leq n$.

Proof. We will use the following formula (the product rule). Let $b=\left(b_{1}, \ldots, b_{n}\right)$ and $\zeta=\left(\zeta_{1}, \ldots, \zeta_{n}\right) \in T_{b}\left(B^{n}\right)$. Then

$$
d \varphi_{b}(\zeta)=\left(d R_{b_{2} \cdots b_{n}} \zeta_{1}+d L_{b_{1}} d R_{b_{3} \cdots b_{n}} \zeta_{2}+\cdots+d L_{b_{1} \cdots b_{n-1}} \zeta_{n}\right)
$$

Hence

$$
\begin{aligned}
& \left.\left(\varphi^{*} \alpha_{x}\right)\right|_{b}(\zeta)=\left.\alpha_{x}\right|_{b_{1} \cdots b_{n}}\left(d R_{b_{2} \cdots b_{n}} \zeta_{1}+d L_{b_{1}} d R_{b_{3} \cdots b_{n}} \zeta_{2}+\cdots+d L_{b_{1} \cdots b_{n-1}} \zeta_{n}\right) \\
& =\left\langle d R_{b_{1} \cdots b_{n}} x, d R_{b_{2} \cdots b_{n}} \zeta_{1}\right\rangle+\left\langle d R_{b_{1} \cdots b_{n}} x, d L_{b_{1}} d R_{b_{3} \cdots b_{n}} \zeta_{2}\right\rangle+\cdots \\
& +\left\langle d R_{b_{1} \cdots b_{n}} x, d L_{b_{1} \cdots b_{n-1}} \zeta_{n}\right\rangle \\
& =\left\langle d R_{b_{1}} x, \zeta_{1}\right\rangle+\left\langle d R_{b_{2}} A d_{b_{1}^{-1}} x, \zeta_{2}\right\rangle+\cdots+\left\langle d R_{b_{n}} A d_{\left(b_{1} \cdots b_{n-1}\right)^{-1}} x, \zeta_{n}\right\rangle \\
& =\sum_{i=1}^{n} \alpha_{x_{i}}\left(\zeta_{i}\right)
\end{aligned}
$$


Proposition 4.26. $\varphi$ is an equivariant momentum map for the dressing action of $K$ on $B^{n}$.

Proof. To show that $\varphi$ is a momentum map we have to check that

$$
-\left.\pi^{\#} \varphi^{*} \alpha_{x}\right|_{b}=x \cdot\left(b_{1}, \ldots, b_{n}\right)
$$

But $-\pi^{\#} \varphi^{*} \alpha_{x}=\left(-\pi^{\#} \alpha_{x_{1}}, \cdots,-\pi^{\#} \alpha_{x_{n}}\right)$ and the result follows from the previous two lemmas. To show that $\varphi$ is equivariant we have to check that $\varphi\left(k \cdot\left(b_{1}, \ldots, b_{n}\right)\right)=$ $k \cdot b_{1} \ldots b_{n}$. This is obvious from the point of view of polygons.

As a consequence of the above proposition we obtain

THEOREM 4.27. The map $\Phi$ carries the symplectic quotient $\left(\left.\varphi\right|_{B_{r}^{n}}\right)^{-1}(1) / K$ diffeomorphically to the moduli space of $n$-gon linkages $M_{r}$.

REMARK 4.28. We obtain a symplectic structure on $M_{r}$ by transport of structure.

4.4. The bending Hamiltonians. In this section we will compute the Hamiltonian vector fields $X_{f_{j}}$ of the functions

$$
f_{j}(b)=\operatorname{tr}\left(\left(b_{1} \cdots b_{j}\right)\left(b_{1} \cdots b_{j}\right)^{*}\right), 1 \leq j \leq n .
$$

Throughout the rest of the paper, we will assume $G=S L_{2}(\mathbb{C})$. Then $G=B K$, where $B=\left\{\left(\begin{array}{cc}a & z \\ 0 & a^{-1}\end{array}\right) \in S L_{2}(\mathbb{C}) \mid a \in \mathbb{R}_{+}, z \in \mathbb{C}\right\}$ and $K=S U(2)$.

We will use the following notation. If $A \in M_{m}(\mathbb{C})$ then $A^{0}=A-\frac{1}{m} \operatorname{tr}(A) I$ will be its projection to the traceless matrices.

Theorem 4.29. Define $F_{j}: B^{n} \rightarrow \mathfrak{k}$ for $b=\left(b_{1}, b_{2}, \ldots, b_{n}\right)$ by

$$
F_{j}(b)=\sqrt{-1}\left[\left(b_{1} \cdots b_{j}\right)\left(b_{1} \cdots b_{j}\right)^{*}\right]^{0}
$$

Then $X_{f_{j}}(b)=\left(F_{j}(b) \cdot\left(b_{1}, \ldots, b_{j}\right), 0, \ldots, 0\right)$ where $\cdot$ is the infinitesimal dressing action of $\mathfrak{k}$ on $B^{j}$, see $\S 4.2$.

Proof. It will be convenient to work on $G^{n}$ and then restrict to $B^{n}$. By the formula for $X_{\varphi}$ of Lemma 4.8 it suffices to compute $D_{i} f_{j}$ and $D_{i}^{\prime} f_{j}$. We recall that

$$
D_{i}^{\prime} \varphi(g)=A d_{g_{i}^{-1}} D_{i} \varphi(g)
$$

hence it suffices to compute $D_{i} f_{j}(g)$. We first reduce to computing $D_{1} f_{j}$ by

Lemma 4.30. $D_{i} f_{j}(g)=A d_{\left(g_{1} \cdots g_{i-1}\right)^{-1}} D_{1} f_{j}(g)$.

Proof. By definition

$$
\left\langle D_{i} f_{j}(g), \nu\right\rangle=\left.\frac{d}{d t}\right|_{t=0} f_{j}\left(g_{1}, \ldots, e^{t \nu} g_{i}, \ldots, g_{n}\right)
$$

But it is elementary that

$$
f_{j}\left(g_{1}, \ldots, e^{t \nu} g_{i}, \ldots, g_{n}\right)=f_{j}\left(\left(A d_{g_{1} \cdots g_{i-1}} e^{t \nu}\right) g_{1}, \ldots, g_{n}\right)
$$


Differentiating at $t=0$ we obtain

$$
\left\langle D_{i} f_{j}(g), \nu\right\rangle=\left\langle D_{1} f_{j}(g), A d_{g_{1} \cdots g_{i-1}} \nu\right\rangle=\left\langle A d_{\left(g_{1} \cdots g_{i-1}\right)^{-1}} D_{1} f_{j}(g), \nu\right\rangle
$$

$\square$

We next have

LEMMA 4.31. $D_{1} f_{j}(g)=F_{j}(g)$.

Proof. By definition

$$
\begin{aligned}
\left\langle D_{1} f_{j}(g), \nu\right\rangle & =\left.\frac{d}{d t}\right|_{t=0} \operatorname{tr}\left[\left(e^{t \nu} g_{1} \cdots g_{j}\right)\left(e^{t \nu} g_{1} \cdots g_{j}\right)^{*}\right] \\
& =\operatorname{tr}\left[\left(\nu g_{1} \cdots g_{j}\right)\left(g_{1} \cdots g_{j}\right)^{*}+\left(g_{1} \cdots g_{j}\right)\left(\nu g_{1} \cdots g_{j}\right)^{*}\right] \\
& =\operatorname{tr}\left[\left(\nu g_{1} \cdots g_{j}\right)\left(g_{1} \cdots g_{j}\right)^{*}\right]+\operatorname{tr}\left[\left(\nu g_{1} \cdots g_{j}\right)\left(g_{1} \cdots g_{j}\right)^{*}\right]^{*} \\
& =\operatorname{tr}\left[\left(\nu g_{1} \cdots g_{j}\right)\left(g_{1} \cdots g_{j}\right)^{*}\right]+\overline{\operatorname{tr}\left[\left(\nu g_{1} \cdots g_{j}\right)\left(g_{1} \cdots g_{j}\right)^{*}\right]} \\
& =2 \operatorname{Retr}\left[\left(\nu g_{1} \cdots g_{j}\right)\left(g_{1} \cdots g_{j}\right)^{*}\right] \\
& =2 \operatorname{Im} \sqrt{-1} \operatorname{tr}\left[\nu\left(g_{1} \cdots g_{j}\right)\left(g_{1} \cdots g_{j}\right)^{*}\right]
\end{aligned}
$$

Since $\nu \in \operatorname{sl}_{2}(\mathbb{C})$ we may replace

$$
\left(g_{1} \cdots g_{j}\right)\left(g_{1} \cdots g_{j}\right)^{*}
$$

by its traceless projection

$$
\left[\left(g_{1} \cdots g_{j}\right)\left(g_{1} \cdots g_{j}\right)^{*}\right]^{0}
$$

Since $t r$ is complex bilinear we obtain

$$
\begin{aligned}
\left\langle D_{1} f_{j}(g), \nu\right\rangle & =2 \operatorname{Imtr}\left(\nu \sqrt{-1}\left[\left(g_{1} \cdots g_{j}\right)\left(g_{1} \cdots g_{j}\right)^{*}\right]^{0}\right) \\
& =\left\langle\sqrt{-1}\left[\left(g_{1} \cdots g_{j}\right)\left(g_{1} \cdots g_{j}\right)^{*}\right]^{0}, \nu\right\rangle .
\end{aligned}
$$

Now we restrict to $B^{n}$ and substitute into our formula for $X_{f_{j}}(b)$ in Lemma 4.8. We obtain

LEMMA 4.32. $\left(X_{f_{j}}\right)_{i}=\left(D_{1} f_{j}(b) \cdot\left(b_{1}, \ldots, b_{n}\right)\right)_{i}=$ the $i$-th component of the infinitesimal dressing action of $D_{1} f_{j}(b) \in \mathfrak{k}$.

Proof. By Lemma 4.8 we have

$$
\begin{gathered}
\left(X_{f_{j}}\right)_{i}=\frac{1}{2}\left[d R_{b_{i}} \mathcal{R} D_{i} f_{j}(b)-d L_{b_{i}} \mathcal{R} D_{i}^{\prime} f_{j}(b)\right] \\
=\frac{1}{2}\left[d R_{b_{i}} \mathcal{R} A d_{\left(b_{1} \cdots b_{i-1}\right)^{-1}} D_{1} f_{j}(b)-d L_{b_{i}} \mathcal{R} A d_{b_{i}^{-1}} A d_{\left(b_{1} \cdots b_{i-1}\right)^{-1}} D_{1} f_{j}(b)\right]
\end{gathered}
$$

We write

$$
A d_{\left(b_{1} \cdots b_{i-1}\right)^{-1}} D_{1} f_{j}(b)=X_{1}+\eta_{1}
$$


with $X_{1} \in \mathfrak{k}$ and $\eta \in \mathfrak{b}$. Hence

$$
\begin{aligned}
& X_{1}=\rho_{\mathfrak{k}}\left(A d_{\left(b_{1} \cdots b_{i-1}\right)^{-1}} D_{1} f_{j}(b)\right) \\
& \eta_{1}=\rho_{\mathfrak{b}}\left(A d_{\left(b_{1} \cdots b_{i-1}\right)^{-1}} D_{1} f_{j}(b)\right)
\end{aligned}
$$

Then

$$
\mathcal{R} A d_{\left(b_{1} \cdots b_{i-1}\right)^{-1}} D_{1} f_{j}(b)=X_{1}-\eta_{1}
$$

We write

$$
A d_{b_{i}^{-1}} X_{1}=X_{2}+\eta_{2}, X_{2} \in \mathfrak{k}, \eta_{2} \in \mathfrak{b}
$$

Then $X_{2}=\rho_{\mathfrak{k}}\left(A d_{b_{i}^{-1}} X_{1}\right), \eta_{2}=\rho_{\mathfrak{b}}\left(A d_{b_{i}^{-1}} X_{1}\right)$ and

$$
A d_{b_{i}^{-1}}\left(X_{1}+\eta_{1}\right)=X_{2}+\eta_{2}+A d_{b_{i}^{-1}} \eta_{1}
$$

Hence

$$
\begin{aligned}
\mathcal{R} A d_{b_{i}^{-1}} A d_{\left(b_{1} \cdots b_{i-1}\right)^{-1}} D_{1} f_{j}(b) & =X_{2}-\eta_{2}-A d_{b_{i}^{-1}} \eta_{1} \\
& =X_{2}+\eta_{2}-2 \eta_{2}-A d_{b_{i}^{-1}} \eta_{1} \\
& =A d_{b_{i}^{-1}} X_{1}-2 \eta_{2}-A d_{b_{i}^{-1}} \eta_{1}
\end{aligned}
$$

Hence

$$
\left(X_{f_{j}}\right)_{i}=\frac{1}{2}\left[d R_{b_{i}} X_{1}-d R_{b_{i}} \eta_{1}-d L_{b_{i}} A d_{b_{i}^{-1}} X_{1}+2 d L_{b_{i}} \eta_{2}+d L_{b_{i}} A d_{b_{i}^{-1}} \eta_{1}\right]
$$

But $d L_{b_{i}} A d_{b_{i}^{-1}}=d R_{b_{i}}$ and we obtain

$$
\left(X_{f_{j}}\right)_{i}=d L_{b_{i}} \eta_{2}
$$

Since $\eta_{2}=\rho_{\mathfrak{b}}\left(A d_{b_{i}^{-1}} \rho_{\mathfrak{k}}\left(A d_{\left(b_{1} \cdots b_{i-1}\right)^{-1}} D_{1} f_{j}(b)\right)\right)$ the lemma follows.

With this Theorem 4.29 is proved.

4.5. Commuting Hamiltonians. In this section we will show the functions

$$
f_{j}(b)=\operatorname{tr}\left(\left(b_{1} \cdots b_{j}\right)\left(b_{1} \cdots b_{j}\right)^{*}\right), 1 \leq j \leq n
$$

Poisson commute. The proof is due to Hermann Flaschka.

Proposition 4.33. $\left\{f_{j}, f_{k}\right\}=0$ for all $j, k$.

Proof. Again we will work on $G^{n}$ and then restrict to $B^{n}$. Without loss of generality we let $j \leq k$.

Recall from Lemma 4.30

$$
D_{i} f_{j}(g)=A d_{\left(g_{1} \cdots g_{i-1}\right)^{-1}} D_{1} f_{j}(g) .
$$

It is easily seen that

$$
D_{i} f_{j}(g)=D_{i-1}^{\prime} f_{j}(g), \text { for } 1 \leq i \leq j .
$$


We now have,

$$
\begin{aligned}
\left\{f_{j}, f_{k}\right\}(g) & =\frac{1}{2} \sum_{i=1}^{n}\left[\left\langle\mathcal{R} D_{i}^{\prime} f_{j}(g), D_{i}^{\prime} f_{k}(g)\right\rangle-\left\langle\mathcal{R} D_{i} f_{j}(g), D_{i} f_{k}(g)\right\rangle\right] \\
& =\frac{1}{2} \sum_{i=1}^{j}\left[\left\langle\mathcal{R} D_{i}^{\prime} f_{j}(g), D_{i}^{\prime} f_{k}(g)\right\rangle-\left\langle\mathcal{R} D_{i} f_{j}(g), D_{i} f_{k}(g)\right\rangle\right] \\
& =\frac{1}{2}\left[\left\langle\mathcal{R} D_{j}^{\prime} f_{j}(g), D_{i}^{\prime} f_{k}(g)\right\rangle-\left\langle\mathcal{R} D_{1} f_{j}(g), D_{1} f_{k}(g)\right\rangle\right] \\
& =\frac{1}{2}\left[\left\langle\mathcal{R} A d_{\left(g_{1} \cdots g_{j}\right)^{-1}} D_{1} f_{j}(g), A d_{\left(g_{1} \cdots g_{j}\right)^{-1}} D_{1} f_{k}(g)\right\rangle-\left\langle\mathcal{R} D_{1} f_{j}(g), D_{1} f_{k}(g)\right\rangle\right] \\
& =\frac{1}{2}\left\langle\mathcal{R} A d_{\left(g_{1} \cdots g_{j}\right)^{-1}} D_{1} f_{j}(g), A d_{\left(g_{1} \cdots g_{j}\right)^{-1}} D_{1} f_{k}(g)\right\rangle
\end{aligned}
$$

since $D_{1} f_{i}(g) \in \mathfrak{k}$ for all $i$. The proposition follows if we can show

$$
\left\langle\mathcal{R} A d_{\left(g_{1} \cdots g_{j}\right)^{-1}} D_{1} f_{j}(g), A d_{\left(g_{1} \cdots g_{j}\right)^{-1}} D_{1} f_{k}(g)\right\rangle=0 .
$$

It follows from the proof of Theorem 4.29 that

$$
\begin{aligned}
A d_{\left(g_{1} \cdots g_{j}\right)^{-1}} D_{1} f_{j}(g) & =\sqrt{-1} A d_{\left(g_{1} \cdots g_{j}\right)^{-1}}\left[\left(g_{1} \cdots g_{j}\right)\left(g_{1} \cdots g_{j}\right)^{*}\right]^{0} \\
& =\sqrt{-1}\left[\left(g_{1} \cdots g_{j}\right)^{*}\left(g_{1} \cdots g_{j}\right)\right]^{0} \in \mathfrak{k} .
\end{aligned}
$$

Hence,

$$
\begin{aligned}
\left\{f_{j}, f_{k}\right\}(g) & =\left\langle\mathcal{R} A d_{\left(g_{1} \cdots g_{j}\right)^{-1}} D_{1} f_{j}(g), A d_{\left(g_{1} \cdots g_{j}\right)^{-1}} D_{1} f_{k}(g)\right\rangle \\
& =\left\langle A d_{\left(g_{1} \cdots g_{j}\right)^{-1}} D_{1} f_{j}(g), A d_{\left(g_{1} \cdots g_{j}\right)^{-1}} D_{1} f_{k}(g)\right\rangle \\
& =\left\langle D_{1} f_{j}(g), D_{1} f_{k}(g)\right\rangle \\
& =0
\end{aligned}
$$

since $\langle$,$\rangle is A d$-invariant. This proves the proposition on $G^{n}$. The result then holds when we restrict to $B^{n}$.

4.6. The Hamiltonian flow. In this section we compute the Hamiltonian flow, $\varphi_{k}^{t}$, associated to $f_{k}$.

Recall from Theorem 4.29 the Hamiltonian field for $f_{k}$ is given by $X_{f_{j}}(b)=$ $\left(F_{j}(b) \cdot\left(b_{1}, \ldots, b_{j}\right), 0, \ldots, 0\right)$ where $\cdot$ is the infinitesimal dressing action of $K$ on $B^{n}$. We now need to solve the system of ordinary differential equations

$$
(*)\left\{\begin{array}{l}
\frac{d b_{i}}{d t}=\left(F_{j}(b) \cdot\left(b_{1}, \ldots, b_{j}\right)\right)_{i}, 1 \leq i \leq j \\
\frac{d b_{i}}{d t}=0, j+1 \leq i \leq n
\end{array}\right.
$$

LEMMA 4.34. $D_{1} f_{j}(b)=F_{j}(b)$ is invariant along solution curves of $\left(^{*}\right)$.

Proof. It suffices to show $\varphi_{j}(b)=b_{1} \cdots b_{j}$ is constant along solution curves. Let $b(t)=\left(b_{1}(t), \ldots, b_{n}(t)\right)$ be a solution of $X_{f_{j}}$. Then 


$$
\begin{aligned}
& \frac{d}{d t} \varphi_{j}(b(t))=\frac{d b_{1}}{d t}(t) b_{2}(t) \cdots b_{j}(t)+b_{1}(t) \frac{d b_{2}}{d t}(t) \cdots b_{j}(t)+\cdots+b_{1}(t) b_{2}(t) \cdots \frac{d b_{j}}{d t}(t) \\
& =\frac{1}{2}\left[\left(\mathcal{R} D_{1} f_{j}(b(t))\right) b_{1}(t)-b_{1}(t) \mathcal{R} D_{1}^{\prime} f_{j}(b(t))\right] b_{2}(t) \cdots b_{j}(t) \\
& +b_{1}(t) \frac{1}{2}\left[\left(\mathcal{R} D_{2} f_{j}(b(t))\right) b_{2}(t)-b_{2}(t) \mathcal{R} D_{2}^{\prime} f_{j}(b(t))\right] b_{3}(t) \cdots b_{j}(t)+\cdots+ \\
& +b_{1}(t) b_{2}(t) \cdots b_{j-1}(t) \frac{1}{2}\left[\left(\mathcal{R} D_{j} f_{j}(b(t))\right) b_{j}(t)-b_{j}(t) \mathcal{R} D_{j}^{\prime} f_{j}(b(t))\right] \\
& =\frac{1}{2}\left[\mathcal{R}\left(D_{1} f_{j}(b(t))\right) b_{1}(t) \cdots b_{j}(t)-b_{1}(t) \cdots b_{j}(t) \mathcal{R}\left(D_{j}^{\prime} f_{j}(b(t))\right)\right] \\
& =\frac{1}{2}\left[\left(D_{1} f_{j}(b(t))\right) b_{1}(t) \cdots b_{j}(t)-b_{1}(t) \cdots b_{j}(t)\left(D_{j}^{\prime} f_{j}(b(t))\right)\right] \\
& =\frac{1}{2}\left[\left(D_{1} f_{j}(b(t))\right) b_{1}(t) \cdots b_{j}(t)-b_{1}(t) \cdots b_{j}(t)\left(A d_{\left(b_{1} \cdots b_{j}\right)^{-1}} D_{1} f_{j}(b(t))\right)\right] \\
& =\frac{1}{2}\left[\left(D_{1} f_{j}(b(t))\right) b_{1}(t) \cdots b_{j}(t)-\left(D_{1} f_{j}(b(t))\right) b_{1}(t) \cdots b_{j}(t)\right]=0
\end{aligned}
$$

Thus $\varphi(b)$ is constant along solution curves of $X_{f_{j}}$, proving the lemma.

REMARK 4.35. It also follows from the previous proof that $f_{j}(b)$ is constant along solution curves of $\left(^{*}\right)$. that

Let $b=\left(\begin{array}{cc}a & z \\ 0 & a^{-1}\end{array}\right)$ with $a \in \mathbb{R}_{+}$and $z \in \mathbb{C}$, then it follows from a simple calculation

$$
\operatorname{det}\left(F_{1}(b)\right)=\frac{1}{4}\left(a^{4}+a^{-4}+|z|^{4}-2+2 a^{2}|z|^{2}+2 a^{-2}|z|^{2}\right) .
$$

Since $a>0$ we see that $a^{4}+a^{-4} \geq 2$ with equality if $a=1$. Therefore, $\operatorname{det}\left(F_{1}(b)\right) \geq 0$ with equality iff $b=1$. From the above argument it follows that $\operatorname{det}\left(F_{j}(b)\right) \geq 0$ with equality iff $b_{1} \cdots b_{j}=1$.

It is also an easy calculation to show

$$
\operatorname{det}\left(F_{j}(b)\right)=\frac{1}{4} f_{j}(b)^{2}-1, \quad \forall b \in B^{n}
$$

LEMMA 4.36. The curve $\exp \left(t F_{j}(b)\right)$ is periodic with period $2 \pi / \sqrt{\frac{1}{4} f_{j}(b)^{2}-1}$

Proof. To simplify notation, let $X=F_{j}(b) \in \mathfrak{k}$. Then

$$
X^{-1}=-\frac{1}{\operatorname{det}(X)} X
$$

giving us

$$
X^{2}=-(\operatorname{det}(X)) X^{-1} X=-\operatorname{det}(X) I
$$


So,

$$
\begin{aligned}
\exp t X & =\sum_{n=0}^{\infty} \frac{t^{n} X^{n}}{n !} \\
& =\sum_{n=1}^{\infty} \frac{(-1)^{n}(t \operatorname{det}(X))^{n}}{(2 n) !} I+\sum_{n=1}^{\infty} \frac{(-1)^{n}(t \operatorname{det}(X))^{n}}{(2 n+1) !} \frac{X}{\sqrt{\operatorname{det}(X)}} \\
& =\cos (t \sqrt{\operatorname{det}(X)}) I+\frac{\sin (t \sqrt{\operatorname{det}(X)})}{\sqrt{\operatorname{det}(X)}} X \\
& =\cos \left(t \sqrt{\frac{1}{4} f_{j}(b)^{2}-1}\right) I+\frac{\sin \left(t \sqrt{\frac{1}{4} f_{j}(b)^{2}-1}\right)}{\sqrt{\frac{1}{4} f_{j}(b)^{2}-1}} F_{j}(b)
\end{aligned}
$$

Therefore the curve is periodic with period $2 \pi / \sqrt{\frac{1}{4} f_{j}(b)^{2}-1}$.

We can now find a solution to the system $(*)$

Proposition 4.37. Suppose $P \in M_{r}$ has vertices given by $b_{1}, \ldots, b_{n}$. Then $P(t)=\varphi_{k}^{t}(P)$ has vertices given by $b_{1}(t), \ldots b_{n}(t)$ where

$$
\begin{gathered}
b_{i}(t)=\left(\exp \left(t F_{j}(b)\right) \cdot\left(b_{1}, \ldots, b_{j}\right)\right)_{i}, 1 \leq i \leq k \\
b_{i}(t)=b_{i}, k+1 \leq i \leq n .
\end{gathered}
$$

Here $\cdot$ is the dressing action of $K$ on $B^{j}$.

Proof. This follows from $F_{j}(b)$ being constant on solution curves of $\left({ }^{*}\right)$. We can see immediately that the $b_{i}$ 's are solutions curves of our system of ordinary differential equations.

Corollary 4.38. The flow $\varphi_{k}^{t}(P)$ is periodic with period $2 \pi / \sqrt{\frac{1}{4} f_{j}(b)^{2}-1}$.

REMARK 4.39. If the $k$-th diagonal is degenerate $\left(b_{1} \cdots b_{k}=1\right)$ then $P$ is a fixed point of $\varphi_{k}^{t}$. In this case the flow has infinite period.

Let $\ell_{k}(b)=2 \cosh ^{-1}\left(\frac{1}{2} f_{k}(b)\right)$, then

$$
d \ell_{k}=\frac{1}{\sqrt{\frac{1}{4} f_{k}(b)^{2}-1}} d f_{k}
$$

and consequently

$$
X_{\ell_{k}}=X_{f_{k}} / \sqrt{\frac{1}{4} f_{k}^{2}-1}
$$

where $X_{\ell_{k}}$ is the Hamiltonian vector field associated to $\ell_{k}$. Since $f_{j}$ is a constant of motion, $X_{\ell_{k}}$ is constant along solutions of $\left(^{*}\right)$ as well. Let $\Psi_{k}^{t}$ be the flow of $X_{\ell_{k}}$. We have the following 
PRoposition 4.40. Suppose $P \in M_{r}^{\prime}$ has vertices $b_{1}, \ldots, b_{n}$. Then $P(t)=\Psi_{k}^{t}(P)$ has vertices $b_{1}(t), \ldots, b_{n}(t)$ given by

$$
\begin{gathered}
\left.b_{i}(t)=\exp \left(\left(t F_{j}(b)\right) / \sqrt{\frac{1}{4} f_{k}(b)^{2}-1}\right) \cdot\left(b_{1}, \ldots, b_{j}\right)\right)_{i}, 1 \leq i \leq k \\
b_{i}(t)=b_{i}, k+1 \leq i \leq n
\end{gathered}
$$

where is the dressing action of $K$ on $B^{n}$.

Thus $\Psi_{k}^{t}$ is periodic with period $2 \pi$ and rotates a part of $P$ around the $k$-th diagonal with constant angular velocity 1 and leaves the other part fixed.

4.7. Angle variables, the momentum polyhedron and a new proof of involutivity. We continue to assume that our n-gons are triangulated by the diagonals $\left\{d_{1 i}, 3 \leq i \leq n-1\right\}$. We assume $P \in M_{r}^{o}$ so none of the $n-2$ triangles, $\triangle_{1}, \Delta_{2}, \ldots, \Delta_{n-2}$, created by the above diagonals are degenerate. We construct a polyhedral surface $S$ bounded by $P$ by filling in the triangles $\Delta_{1}, \Delta_{2}, \ldots, \Delta_{n-2}$. Hence, $\triangle_{1}$ has edges $e_{1}, e_{2}$, and $d_{13}, \triangle_{2}$ has edges $d_{13}, e_{3}$, and $d_{14}, \ldots$, and $\triangle_{n-2}$ has edges $d_{1, n-1}, e_{n-1}$, and $e_{n}$.

We define $\hat{\theta}_{i}$ to be the oriented dihedral angle measured from $\Delta_{i}$ to $\Delta_{i+1}, 1 \leq$ $i \leq n-3$. We define the $i$-th angle variable $\theta_{i}$ by

$$
\theta_{i}=\pi-\hat{\theta}_{i}, 1 \leq i \leq n-3 .
$$

THEOREM 4.41. $\left\{\theta_{1}, \ldots, \theta_{n-3}\right\}$ are angle variables, that is we have

(i) $\left\{\ell_{i}, \theta_{j}\right\}=\delta_{i j}$

(ii) $\left\{\theta_{i}, \theta_{j}\right\}=0$.

Proof. The proof is identical to that of [KM2, §4].

We next describe the momentum polyhedron $B_{r}$ for the action of the above (n$3)$-torus by bendings. Hence,

$$
B_{r}=\left\{\ell\left(M_{r}\right) \subset\left(\mathbb{R}_{\geq 0}\right)^{n-3}: \ell=\left(\ell_{1}, \ldots, \ell_{n-3}\right)\right\} .
$$

Let $\left(\ell_{1}, \ldots, \ell_{n-3}\right) \in\left(\mathbb{R}_{\geq 0}\right)^{n-3}$ be given. We first consider the problem of constructing the triangles, $\Delta_{1}, \triangle_{2}, \ldots, \Delta_{n-2}$ above. We note that there are three triangle inequalities $E_{i}(\ell, r), 1 \leq i \leq n-2$, among the $r_{i}$ 's and $\ell_{j}$ 's that give necessary and sufficient conditions for the existence of $\triangle_{i}$. Once we have obtained the triangles $\triangle_{1}, \Delta_{2}, \ldots, \Delta_{n-2}$, we can glue them along the diagonals $d_{1 i}, 3 \leq i \leq n-1$, and obtain a polyhedron surface $S$ and a n-gon $P$. We obtain

THEOREM 4.42. The momentum polyhedron $B_{r} \subset\left(\mathbb{R}_{\geq 0}\right)^{n-3}$ is defined by the $3(n-2)$ triangle inequalities

$$
\begin{gathered}
\left|r_{1}-r_{2}\right| \leq \ell_{1} \leq r_{1}+r_{2} \\
\left|\ell_{1}-r_{3}\right| \leq \ell_{2} \leq \ell_{1}+r_{3} \\
\vdots \\
\left|\ell_{n-4}-r_{n-2}\right| \leq \ell_{n-3} \leq \ell_{n-4}+r_{n-2} \\
\left|r_{n-1}-r_{n}\right| \leq \ell_{n-3} \leq r_{n-1}+r_{n}
\end{gathered}
$$


Here $r=\left(r_{1}, \ldots, r_{n}\right)$ is fixed, the $\ell_{i}$ 's, $1 \leq i \leq n-3$, are the variables.

As a consequence we have

THEOREM 4.43. The functions $\ell_{1}, \ell_{2}, \ldots, \ell_{n-3}$ on $M_{r}$ are functionally independent.

The theorem follows from Corollary 4.45. We will apply the next lemma with $M=\ell^{-1}\left(B_{r}^{o}\right)$, the inverse image of the interior of the momentum polyhedron under $\ell=\left(\ell_{1}, \ell_{2}, \ldots, \ell_{n-3}\right)$. Then $M \simeq B_{r}^{o} \times\left(S^{1}\right)^{n-3}$.

LEMMA 4.44. Suppose $M=M_{r}^{o}$ is a connected real-analytic manifold and $F=\left(f_{1}, \ldots, f_{k}\right): M^{n} \rightarrow \mathbb{R}^{k}, n \geq k$, is a real-analytic map such that $F(M)$ contains a $k$-ball. Then the 1-forms $d f_{1}, \ldots, d f_{k}$ are linearly independent over $C^{\infty}(M)$.

Proof. Since the 1-forms $d f_{1}, d f_{2}, \ldots, d f_{k}$ are real-analytic, the set of points $x \in M$ such that $\left.d f_{1}\right|_{x}, \ldots,\left.d f_{k}\right|_{x}$ are not independent over $\mathbb{R}$ is an analytic subset $W$ of $M$. Let $M^{0}=M-W$. Hence either $M^{\circ}$ is empty or it is open and dense. But by Sard's Theorem, $F(W)$ has measure zero. Since $F(M)$ does not have measure zero, $M \neq W$ and $M^{\circ}$ is nonempty, hence open and dense. Therefore, if there exists $\varphi_{1}, \ldots, \varphi_{k} \in C^{\infty}(M)$ such that $\sum_{i=1}^{k} \varphi_{i} d f_{i}=0$ then $\left.\varphi_{i}\right|_{M^{\circ}} \equiv 0,1 \leq i \leq k$, and by density $\varphi_{i} \equiv 0,1 \leq i \leq k$.

CoRollaRy 4.45. The restrictions of $d \ell_{1}, d \ell_{2}, \ldots, d \ell_{n-3}$ to $M \subset M_{r}$ are independent over $C^{\infty}(M)$.

REMARK 4.46. Since $\ell$ is onto, if there exists $\Phi \in C^{\infty}\left(B_{r}\right)$ such that $\Phi\left(\ell_{1}(x), \ldots, \ell_{k}(x)\right) \equiv 0$, then $\Phi \equiv 0$.

We conclude this chapter by giving a second proof that the bending flows on disjoint diagonals commute. Since $M_{r}^{o}$ is dense in $M_{r}$, it suffices to prove

LEMMA 4.47. $\Psi_{i}^{s}\left(\Psi_{j}^{t}(P)\right)=\Psi_{j}^{t}\left(\Psi_{i}^{s}(P)\right)$, for $P \in M_{r}^{o}$.

Proof. We assume $i>j$. We observe that the diagonals $d_{1 i}$ and $d_{1 j}$ divide the surface $S$ into three polyhedral "flaps", $I, I I, I I I$ (the boundary of $I$ contains $e_{1}$, the boundary of $I I$ contains $e_{i}$, and the boundary of $I I I$ contains $e_{j}$ ). Let $R_{i}^{s}$ and $R_{j}^{t}$ be the one parameter groups of rotations around $d_{1 i}$ and $d_{1 j}$, respectively. We first record what $\Psi_{i}^{s} \circ \Psi_{j}^{t}$ does to the flaps.

$$
\begin{aligned}
& \Psi_{i}^{s} \circ \Psi_{j}^{t}(I)=R_{i}^{s} R_{j}^{t}(I) \\
& \Psi_{i}^{s} \circ \Psi_{j}^{t}(I I)=R_{i}^{s}(I I) \\
& \Psi_{i}^{s} \circ \Psi_{j}^{t}(I I I)=I I I
\end{aligned}
$$

Now we compute what $\Psi_{j}^{t} \circ \Psi_{i}^{s}$ does to the flaps. The point is, after the bending on $d_{1 i}$, the diagonal $d_{1 j}$ moves $R_{i}^{s} d_{1 j}$. Hence, the next bending rotates $I$ around $R_{i}^{s} d_{1 j}$. Hence, the next bending curve is $R_{i}^{s} \circ R_{j}^{t} \circ R_{i}^{-s}$. We obtain

$$
\begin{aligned}
& \Psi_{j}^{t} \circ \Psi_{i}^{s}(I)=\left(R_{i}^{s} R_{j}^{t} R_{i}^{-s}\right) R_{i}^{s}(I) \\
& \Psi_{j}^{t} \circ \Psi_{i}^{s}(I I)=R_{i}^{s}(I I) \\
& \Psi_{j}^{t} \circ \Psi_{i}^{s}(I I I)=I I I .
\end{aligned}
$$


5. Symplectomorphism of $M_{r}\left(\mathbb{E}^{3}\right)$ and $M_{r}\left(\mathbb{H}^{3}\right)$. Recall $r$ is not on a wall of $D_{n}$. Then by Theorem 3.2 of this paper, the hyperbolic Gauss map $\gamma=\gamma_{h}$ : $M_{r}\left(\mathbb{H}^{3}\right) \rightarrow Q_{s s t}(r)$ is a diffeomorphism. Moreover by Theorem 2.3 of [KM2], the Euclidean Gauss map $\gamma_{e}: M_{r}\left(\mathbb{E}^{3}\right) \rightarrow Q_{s s t}(r)$ is also a diffeomorphism. We obtain

TheOREM 5.1. Suppose $r$ is not on a wall of $D_{r}$, then the composition $\gamma_{h}^{-1} \circ \gamma_{e}$ : $M_{r}\left(\mathbb{E}^{3}\right) \rightarrow M_{r}\left(\mathbb{H}^{3}\right)$ is a diffeomorphism.

REMARK 5.2. The result that $M_{r}\left(\mathbb{E}^{3}\right)$ and $M_{r}\left(\mathbb{H}^{3}\right)$ are (noncanonically) diffeomorphic was obtained by [Sa].

It does not appear to be true that $\gamma_{h}^{-1} \circ \gamma_{e}$ is a symplectomorphism.

5.1. A Formula of Lu. In the next several sections we will prove that $M_{r}\left(\mathbb{H}^{3}\right)$ is symplectomorphic to $M_{r}\left(\mathbb{E}^{3}\right)$.

We first define a family of nondegenerate Poisson structures $\pi_{\epsilon}, \epsilon \in[0,1]$, on the 2-sphere, $S^{2} \simeq K / T$. Letting $\omega_{\epsilon}$ be the corresponding family of symplectic forms we show the cohomology classes $\left[\omega_{\epsilon}\right]$ of $\omega_{\epsilon}$ in $H^{2}\left(S^{2}\right)$ are constant.

Fix $\lambda \in \mathbb{R}_{+}$and $\Lambda=X \wedge Y \in \wedge^{2} \mathfrak{k}$, where $X=\frac{1}{2}\left(\begin{array}{cc}0 & 1 \\ -1 & 0\end{array}\right)$ and $Y=\frac{1}{2}\left(\begin{array}{ll}0 & i \\ i & 0\end{array}\right)$. The following family of Poisson structures $\pi_{\epsilon}$ on $K / T \simeq S^{2}$ for $\epsilon \in(0,1]$ are due to J.-H. $\mathrm{Lu}[\mathrm{Lu} 2]$.

$$
\pi_{\epsilon}=\epsilon\left[\pi_{\infty}-\tau(\epsilon) \pi_{0}\right]
$$

where $\pi_{\infty}=p_{*} \pi_{K}=p_{*}\left(d L_{k} \Lambda-d R_{k} \Lambda\right), \tau(\epsilon)=\frac{1}{1-e^{4 \epsilon \lambda}}$, and $\pi_{0}=2 d L_{k} \Lambda$. Here $p: K \rightarrow K / T$ is the projection map. Then

$$
\pi_{\epsilon}(k)=\epsilon\left(d L_{k} \Lambda-d R_{k} \Lambda\right)-\frac{2 \epsilon}{1-e^{4 \epsilon \lambda}} d L_{k} \Lambda
$$

LEMMA 5.3.

$$
\lim _{\epsilon \rightarrow 0} \pi_{\epsilon}=\frac{1}{4 \lambda} \pi_{0}
$$

Proof. The proof of the lemma is a simple application of L'Hôpital's rule.

LEMMA 5.4. $\pi_{\epsilon}$ is nondegenerate for $\epsilon \in[0,1]$.

We will prove Lemma 5.4 in Proposition 5.23, where we show $\left(K / T, \pi_{\epsilon}\right)$ is symplectomorphic to a symplectic leaf of the Poisson Lie group $\left(B_{\epsilon}, \widehat{\pi}_{B_{\epsilon}}\right)$

We leave it to the reader to verify the Poisson structures on $S^{2}$ can be written

$$
\pi_{\infty}=\frac{1}{2}\left(1+\alpha^{2}+\beta^{2}\right) \frac{\partial}{\partial \alpha} \wedge \frac{\partial}{\partial \beta} .
$$

and

$$
\pi_{0}=\frac{1}{2}\left(1+\alpha^{2}+\beta^{2}\right)^{2} \frac{\partial}{\partial \alpha} \wedge \frac{\partial}{\partial \beta}
$$

where $(\alpha, \beta)$ are coordinates obtained by stereographic projection with respect to the north pole (see [LW]). $\pi_{\infty}$ is the Bruhat-Poisson structure on $K / T$. We now 
let $\omega_{\epsilon}$ be the symplectic form obtained by inverting $\pi_{\epsilon}$ (this is possible since $\pi_{\epsilon}$ is nondegenerate).

$$
\omega_{\epsilon}=\frac{-d \alpha \wedge d \beta}{\epsilon\left(\frac{1}{2}\left(1+\alpha^{2}+\beta^{2}\right)-\frac{1}{2} \tau(\epsilon)\left(1+\alpha^{2}+\beta^{2}\right)^{2}\right)}, \epsilon \in(0,1]
$$

Let $\omega_{0}$ be the limiting symplectic structure

$$
\omega_{0}=-8 \lambda \frac{d \alpha \wedge d \beta}{\left(1+\alpha^{2}+\beta^{2}\right)^{2}}
$$

LEMMA 5.5.

$$
\int_{\mathbb{R}^{2}} \omega_{0}=-8 \pi \lambda
$$

Proof.

$$
\begin{aligned}
\int_{\mathbb{R}^{2}} \omega_{0} & =\int_{\mathbb{R}^{2}}-8 \lambda \frac{d \alpha \wedge d \beta}{\left(1+\alpha^{2}+\beta^{2}\right)^{2}} \\
& =-8 \lambda \int_{\theta=0}^{\theta=2 \pi} \int_{r=0}^{r=\infty} \frac{r d r \wedge d \theta}{\left(1+r^{2}\right)^{2}} \\
& =-16 \pi \lambda \int_{u=1}^{u=\infty} \frac{(1 / 2) d u}{u^{2}} \\
& =-8 \pi \lambda
\end{aligned}
$$

LEMMA 5.6.

$$
\int_{\mathbb{R}^{2}} \omega_{\epsilon}=-8 \pi \lambda, \epsilon \in(0,1]
$$

Proof. Note that $\tau(\epsilon)<0$. Then

$$
\begin{aligned}
\int_{\mathbb{R}^{2}} \omega_{\epsilon} & =-\frac{2}{\epsilon} \int_{\mathbb{R}^{2}} \frac{d \alpha \wedge d \beta}{\left(1+\alpha^{2}+\beta^{2}\right)-\tau(\epsilon)\left(1+\alpha^{2}+\beta^{2}\right)^{2}} \\
& =-\frac{2}{\epsilon} \int_{\theta=0}^{\theta=2 \pi} \int_{r=0}^{r=\infty} \frac{r d r \wedge d \theta}{\left(1+r^{2}\right)-\tau(\epsilon)\left(1+r^{2}\right)^{2}} \\
& =-\frac{4 \pi}{\epsilon} \int_{u=1}^{u=\infty} \frac{(1 / 2) d u}{\left.u-\tau(\epsilon) u^{2}\right)} \\
& =-\frac{2 \pi}{\epsilon} \log \left|\frac{\tau(\epsilon)-1}{\tau(\epsilon)}\right| \\
& =-\frac{2 \pi}{\epsilon} \log \left(e^{4 \epsilon \lambda}\right) \\
& =-8 \pi \lambda
\end{aligned}
$$

We have proved the following

LEMma 5.7. The cohomology classes $\left[\omega_{\epsilon}\right]$ of $\omega_{\epsilon}$ in $H^{2}\left(S^{2}\right)$ are constant.

REMARK 5.8. The previous lemma is a special case of Lemma 5.1 of [GW]. 
5.2. Symplectomorphism of $\left(\Sigma_{\lambda}(\epsilon), \pi_{B_{\epsilon}}\right)$ and $\left(K / T, \pi_{\lambda, \epsilon}\right)$. In this section we obtain the Poisson structure $\pi_{\epsilon}$ from a deformed Manin triple $\left(\mathfrak{g}_{\epsilon}, \mathfrak{k}, \mathfrak{b}_{\epsilon}\right)$.

For $\epsilon>0$, we define the isomorphism $f_{\epsilon}: \mathfrak{g}_{\epsilon} \rightarrow \mathfrak{g}$ by $f_{\epsilon}=\rho_{\mathfrak{k}}+\epsilon \rho_{\mathfrak{b}}$, so that $f_{\epsilon}(X+\xi)=X+\epsilon \xi$ for $X \in \mathfrak{k}$ and $\xi \in \mathfrak{b}$. We will define a Lie bracket on $\mathfrak{g}_{\epsilon}$ by the pullback of the Lie bracket on $\mathfrak{g},[u, v]_{\epsilon}=f_{1 / \epsilon}\left[f_{\epsilon} u, f_{\epsilon} v\right]$. We also define $\langle,\rangle_{\epsilon}$ as the pullback of $\langle$,$\rangle . Here [,] and \langle$,$\rangle are the usual structures on \mathfrak{g}$. We define $\mathcal{B}_{\epsilon}: \mathfrak{g} \rightarrow \mathfrak{g}^{*}$ as the map induced by $\langle,\rangle_{\epsilon}$. To simplify notation, the subscripts will be dropped when $\epsilon=1$.

The following lemma gives us a formula for the Lie bracket on $\mathfrak{g}_{\epsilon}$.

Lemma 5.9. $[X+\alpha, Y+\beta]_{\epsilon}=[X, Y]+\epsilon \rho_{\mathfrak{k}}[X, \beta]+\epsilon \rho_{\mathfrak{k}}[\alpha, Y]+\rho_{\mathfrak{b}}[X, \beta]+\rho_{\mathfrak{b}}[\alpha, Y]+$ $\epsilon[\alpha, \beta]$, where $X, Y \in \mathfrak{k}$ and $\alpha, \beta \in \mathfrak{b}$.

Proof.

$$
\begin{aligned}
{[X+\alpha, Y+\beta]_{\epsilon} } & =f_{1 / \epsilon}\left[f_{\epsilon}(X+\alpha), f_{\epsilon}(Y+\beta)\right] \\
& =f_{1 / \epsilon}[X+\epsilon \alpha, Y+\epsilon \beta] \\
& =f_{1 / \epsilon}\left\{[X, Y]+\epsilon[X, \beta]+\epsilon[\alpha, Y]+\epsilon^{2}[\alpha, \beta]\right\} \\
& =f_{1 / \epsilon}\left\{[X, Y]+\epsilon \rho_{\mathfrak{k}}[X, \beta]+\epsilon \rho_{\mathfrak{b}}[X, \beta]+\epsilon \rho_{\mathfrak{k}}[\alpha, Y]+\epsilon \rho_{\mathfrak{b}}[\alpha, Y]+\epsilon^{2}[\alpha, \beta]\right\} \\
& =[X, Y]+\epsilon \rho_{\mathfrak{k}}[X, \beta]+\epsilon \rho_{\mathfrak{k}}[\alpha, Y]+\rho_{\mathfrak{b}}[X, \beta]+\rho_{\mathfrak{b}}[\alpha, Y]+\epsilon[\alpha, \beta]
\end{aligned}
$$

We leave it to the reader to check

LEMMA 5.10. $\langle,\rangle_{\epsilon}=\epsilon\langle$,

Let $G_{\epsilon}$ be the simply-connected Lie group with Lie algebra $\mathfrak{g}_{\epsilon}$. Let $F_{\epsilon}: G_{\epsilon} \rightarrow G$ be the isomorphism induced by $f_{\epsilon}$. We have a commutative diagram of isomorphisms.

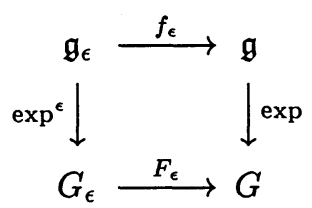

Let $x \in \mathfrak{g}_{\epsilon}$. We use the identity map to identify $\mathfrak{g}$ and $\mathfrak{g}_{\epsilon}$ as vector spaces. In what follows, we will make frequent use of

LEMMA 5.11. $\widehat{A d}\left(\exp ^{\epsilon} x\right)=A d(\exp \epsilon x)$ as elements in $G L(\mathfrak{b})$ for all $x \in \mathfrak{b}_{\epsilon}=\mathfrak{b}$. Here $\widehat{A d}$ denotes the adjoint action of $G_{\epsilon}$ on $\mathfrak{g}_{\epsilon}$.

Proof. By [Wa, pg. 114],

$$
\begin{aligned}
\widehat{A d}\left(\exp ^{\epsilon} x\right) & =e^{\widehat{a d x}} \\
& =e^{\epsilon a d x} \\
& =e^{a d(\epsilon x)} \\
& =A d(\exp \epsilon x)
\end{aligned}
$$


Given our deformed Manin triple on $G_{\epsilon},\left(\mathfrak{g}_{\epsilon}, \mathfrak{k}, \mathfrak{b}_{\epsilon}\right)$, we will construct a Poisson structure $\widehat{\pi}_{B_{\epsilon}}$ on $B_{\epsilon}$, the simply-connected Lie group with Lie algebra, $\mathfrak{b}_{\epsilon}$. We will denote all quantities associated to the deformed Manin triple with a hat ${ }^{\wedge}$. $[\mathrm{LW}]$

We define the Poisson Lie structure on $B_{\epsilon}$ by the Lu-Weinstein Poisson tensor

$$
\widehat{\pi}_{B_{\epsilon}}(b)\left(\widehat{d R}_{b^{-1}}^{*} \alpha_{X}, \widehat{d R}_{b^{-1}}^{*} \alpha_{Y}\right)=\left\langle\rho_{\mathfrak{k}}\left(\widehat{A d}_{b^{-1}} \mathcal{B}_{\epsilon}^{-1}\left(\alpha_{X}\right)\right), \rho_{\mathfrak{b}}\left(\widehat{A d}_{b^{-1}} \mathcal{B}_{\epsilon}^{-1}\left(\alpha_{Y}\right)\right)\right\rangle_{\epsilon}
$$

where $\alpha_{X}, \alpha_{Y} \in \mathfrak{b}_{\epsilon}^{*}, \alpha_{X}=\langle X, \cdot\rangle_{1}$ and $\alpha_{Y}=\langle Y, \cdot\rangle_{1}$.

REMARK 5.12. Since $\lim _{\epsilon \rightarrow 0}\langle,\rangle_{\epsilon}=0$, it appears as if the limiting Poisson structure $\lim _{\epsilon \rightarrow 0} \widehat{\pi}_{B_{\epsilon}}$ will vanish. However, we will see in Proposition 5.14 that the limiting Poisson structure is associated to the Manin triple $\left(\mathfrak{g}_{0}, \mathfrak{k}, \mathfrak{b}_{0}\right)$ and $\langle,\rangle_{0}=\left.\frac{d}{d \epsilon}\right|_{\epsilon=0}\langle,\rangle_{\epsilon}$.

We denote by $\pi_{B_{\epsilon}}$ the Poisson structure on $B_{\epsilon}$ using the scaled bilinear form $\frac{1}{\epsilon}\langle,\rangle_{\epsilon}=\langle$,$\rangle . Then$

$$
\pi_{B_{\epsilon}}(b)\left(\widehat{d R}_{b^{-1}}^{*} \alpha_{X}, \widehat{d R}_{b^{-1}}^{*} \alpha_{Y}\right)=\left\langle\rho_{\mathfrak{k}}\left(\widehat{A d}_{b^{-1}} \mathcal{B}_{1}^{-1}\left(\alpha_{X}\right)\right), \rho_{\mathfrak{b}}\left(\widehat{A d}_{b^{-1}} \mathcal{B}_{1}^{-1}\left(\alpha_{Y}\right)\right)\right\rangle
$$

where $\alpha_{X}, \alpha_{Y} \in \mathfrak{b}_{\epsilon}^{*}$. For the following we will let $X_{\epsilon}=\mathcal{B}_{\epsilon}^{-1}\left(\alpha_{X}\right) \in \mathfrak{k}$, again dropping the subscript when $\epsilon=1$, so that $X_{\epsilon}=\frac{1}{\epsilon} X$.

LEMMA 5.13. $\widehat{\pi}_{B_{\epsilon}}=\frac{1}{\epsilon} \pi_{B_{\epsilon}}$

Proof.

$$
\begin{aligned}
\widehat{\pi}_{B_{\epsilon}}(b)\left(\widehat{d R}_{b^{-1}}^{*} \alpha_{X}, \widehat{d R}_{b^{-1}}^{*} \alpha_{Y}\right) & =\left\langle\rho_{\mathfrak{k}}\left(\widehat{A d}_{b^{-1}} X \epsilon\right), \rho_{\mathfrak{b}}\left(\widehat{A d}_{b^{-1}} Y_{\epsilon}\right)\right\rangle_{\epsilon} \\
& =\left\langle\rho_{\mathfrak{k}}\left(\widehat{A d}_{b^{-1}} \frac{1}{\epsilon} X\right), \rho_{\mathfrak{b}}\left(\widehat{A d}_{b^{-1}} \frac{1}{\epsilon} Y\right)\right\rangle_{\epsilon} \\
& =\frac{1}{\epsilon}\left\langle\rho_{\mathfrak{k}}\left(\widehat{A d}_{b^{-1}} X\right), \rho_{\mathfrak{b}}\left(\widehat{A d}_{b^{-1}} Y\right)\right\rangle
\end{aligned}
$$

Proposition 5.14. $\lim _{\epsilon \rightarrow 0} \widehat{\pi}_{B_{\epsilon}}(b)\left(\widehat{d R}_{b^{-1}}^{*} \alpha_{X}, \widehat{d R}_{b^{-1}}^{*} \alpha_{Y}\right)=-\langle\log b,[X, Y]\rangle$.

Proof.

$$
\begin{aligned}
\lim _{\epsilon \rightarrow 0} \widehat{\pi}_{B_{\epsilon}}(b)\left(\widehat{d R}_{b^{-1}}^{*} \alpha_{X}, \widehat{d R}_{b^{-1}}^{*} \alpha_{Y}\right) & =\lim _{\epsilon \rightarrow 0} \frac{1}{\epsilon}\left\langle\rho_{\mathfrak{k}}\left(\widehat{A d}_{b^{-1}} X\right), \rho_{\mathfrak{b}}\left(\widehat{A d}_{b^{-1}} Y\right)\right\rangle \\
& =\lim _{\epsilon \rightarrow 0} \frac{1}{\epsilon} 2 \operatorname{Im} \operatorname{tr}\left(\rho_{\mathfrak{k}}\left(A d_{e^{-\epsilon \log b}} X\right) \rho_{\mathfrak{b}}\left(A d_{e^{-\epsilon \log b}} Y\right)\right) \\
& =\lim _{\epsilon \rightarrow 0} 2 \operatorname{Im} \operatorname{tr}\left(\rho_{\mathfrak{k}}\left(A d_{e^{-\epsilon} \log b} X\right) \rho_{\mathfrak{b}}(-\log b Y+Y \log b)\right) \\
& =-2 \operatorname{Im} \operatorname{tr}\left(X \rho_{\mathfrak{b}}[\log b, Y]\right) \\
& =-2 \operatorname{Im} \operatorname{tr}(X[\log b, Y]) \\
& =-2 \operatorname{Im} \operatorname{tr}(\log b[X, Y]) \\
& =-\langle\log b,[X, Y]\rangle
\end{aligned}
$$

REMARK 5.15. Before stating the next corollary, note that the limit Lie algebra $\mathfrak{b}_{0}$ is abelian whence the limit Lie group $B_{0}$ is abelian. Hence, $\exp _{0}: \mathfrak{b}_{0}=T_{o}\left(B_{0}\right) \rightarrow B_{0}$ is 
the canonical identification of the vector space $B_{0}$ with its tangent space at the origin. Hence, $\exp _{0}$ is an isomorphism of Lie groups and $\exp _{0}^{*}$ carries invariant 1 -forms on $B_{0}$ to invariant 1 -forms on $\mathfrak{b}_{0}$.

CoRollary 5.16. $\lim _{\epsilon \rightarrow 0} \widehat{\pi}_{B_{\epsilon}}$ is the negative of the Lie Poisson structure on $\mathfrak{k}^{*} \simeq \mathfrak{b}_{0}$ transferred to $B_{0}$ using the exponential map on the vector space $B_{0}$.

Proof. The proof is left to the reader.

REMARK 5.17. $\left(K, \epsilon \pi_{K}\right)$ is the dual Poisson Lie group of $\left(B_{\epsilon}, \widehat{\pi}_{B_{\epsilon}}\right)$.

We will denote the dressing action of $K$ on $B_{\epsilon}$ by $\widehat{D}_{\epsilon}^{\ell}$ and the infinitesimal dressing action of $\mathfrak{k}$ on $B_{\epsilon}$ by $\hat{d}_{\epsilon}^{\ell}$. By definition $\widehat{d}_{\epsilon}^{\ell}(b)(X)=\widehat{\pi}_{B_{\epsilon}}\left(\cdot, \alpha_{X}\right)$. We then have the following.

LEMMA 5.18. $\hat{d}_{\epsilon}^{\ell}(b)(X)=\frac{1}{\epsilon} d^{\ell}(b)(X)$

Proof. Follows immediately from Lemma 5.13 and the definition of dressing action.

REMARK 5.19. $\lim _{\epsilon \rightarrow 0} \hat{d}_{\epsilon}^{\ell}(b)(X)=a d^{*}(X)(\log b)$

For the remainder of the section, fix $\lambda \in \mathbb{R}_{+}$and $a=\exp ^{\epsilon} \lambda H \in B_{\epsilon}$, where $H=\operatorname{diag}(1,-1) \in \mathfrak{a}_{\epsilon}$. Let $\varphi_{\epsilon}: K \rightarrow \Sigma_{\lambda}^{\epsilon} \subset B_{\epsilon}$ be the map defined by $\varphi_{\epsilon}(k)=$ $\widehat{D}_{\epsilon}^{\ell}(k)(a)=\rho_{B_{\epsilon}}(k * a)$, where $\Sigma_{\lambda}^{\epsilon}$ is the symplectic leaf through the point $a \in B_{\epsilon}$. The map $\varphi_{\epsilon}$ induces a diffeomorphism from $K / T$ onto $\Sigma_{\lambda}^{\epsilon}$ which we will also denote by $\varphi_{\epsilon}$. Recall the family of Poisson tensors on $K / T$ given in $\S 5.1$

$$
\pi_{\lambda, \epsilon}=\epsilon\left(\pi_{\infty}-\tau(\epsilon \lambda) \pi_{0}\right)
$$

LeMma 5.20. The map $\varphi_{\epsilon}: K / T \rightarrow B_{\epsilon}$ is $K$-equivariant, where $\left(K, \epsilon \pi_{K}\right)$ acts on $\left(K / T, \pi_{\lambda, \epsilon}\right)$ by left multiplication and $B_{\epsilon}$ by the dressing action.

Proof. $\varphi_{\epsilon}(g \cdot k)=\widehat{D}_{\epsilon}^{\ell}(g k)(a)=\widehat{D}_{\epsilon}^{\ell}(g)\left(\widehat{D}_{\epsilon}^{\ell}(k)(a)\right)=g \cdot \varphi_{\epsilon}(k)$.

REMARK 5.21. The action of $\left(K, \epsilon \pi_{K}\right)$ on $\left(K / T, \pi_{\lambda, \epsilon}\right)$ by left multiplication is a Poisson action.

Since $K / T$ is a symplectic manifold, there is a momentum map for the action of $K$ on $K / T$, see [Lu1, Theorem 3.16]. We will see as a consequence of Proposition 5.23

LEMMA 5.22. The momentum map for the action of $\left(K, \epsilon \pi_{K}\right)$ on $\left(K / T, \pi_{\lambda, \epsilon}\right)$ is $\varphi_{\epsilon}$.

Proposition 5.23. The map $\varphi_{\epsilon}$ induces a symplectomorphism from $\left(K / T, \pi_{\lambda, \epsilon}\right)$ to $\left(\Sigma_{\lambda}^{\epsilon}, \widehat{\pi}_{B_{\epsilon}}\right)$.

Proof. Since the $K$-actions on $K / T$ and $\Sigma_{\lambda}^{\epsilon}$ are Poisson and the map $\varphi_{\epsilon}: K / T \rightarrow$ $\Sigma_{\lambda}^{\epsilon}$ is a $K$-equivariant diffeomorphism, if $\left(d \varphi_{\epsilon}\right)_{e}\left(\pi_{\lambda, \epsilon}(e)\right)=\widehat{\pi}_{B_{\epsilon}}(a)$ then it follows that $\left(d \varphi_{\epsilon}\right)_{k}\left(\pi_{\lambda, \epsilon}(k)\right)=\widehat{\pi}_{B_{\epsilon}}(\varphi(k))$ for all $k \in K / T$. 
We will need the following lemmas to prove the proposition. We let $E=\left(\begin{array}{ll}0 & 1 \\ 0 & 0\end{array}\right) \in \mathfrak{b}$ and $\Lambda=E \wedge i E \in \mathfrak{b} \wedge \mathfrak{b}$. If we set $\widehat{\pi}_{\Lambda}(b)=\frac{1}{\epsilon}\left(\widehat{d L}_{b} \Lambda-\widehat{d R}_{b} \Lambda\right)$, we then have the following.

LEMMA 5.24. $\left.\widehat{\pi}_{B_{\epsilon}}\right|_{a}=\left.\frac{1}{2} \widehat{\pi}_{\Lambda}\right|_{a}$ for $a=\exp ^{\epsilon} \lambda H$.

Proof. Let $X=\left(\begin{array}{cc}s i & u \\ -\bar{u} & -s i\end{array}\right), Y=\left(\begin{array}{cc}t i & v \\ -\bar{v} & -t i\end{array}\right) \in \mathfrak{k}$

$$
\begin{aligned}
\widehat{\pi}_{B_{\epsilon}}(a)\left(\widehat{d R}_{a^{-1}}^{*} \alpha_{X}, \widehat{d R}_{a^{-1}}^{*} \alpha_{Y}\right) & =\frac{1}{\epsilon}\left\langle\rho_{\mathfrak{k}}\left(\widehat{A d}_{a^{-1}} X\right), \rho_{\mathfrak{b}}\left(\widehat{A d}_{a^{-1}} Y\right)\right\rangle \\
& =\frac{1}{\epsilon}\left\langle X, \widehat{A d}_{a} \rho_{\mathfrak{b}}\left(\widehat{A d}_{a^{-1}} Y\right)\right\rangle
\end{aligned}
$$

We can see,

$$
\begin{aligned}
\widehat{A d}_{a} \rho_{\mathfrak{b}}\left(\widehat{A d}_{a^{-1}} Y\right) & =\widehat{A d}_{a} \rho_{\mathfrak{b}}\left(\widehat{A d}_{a^{-1}}\left(\begin{array}{cc}
t i & v \\
-\bar{v} & -t i
\end{array}\right)\right) \\
& =\widehat{A d}_{a} \rho_{\mathfrak{b}}\left(\begin{array}{cc}
t i & e^{-2 \epsilon \lambda} v \\
-e^{2 \epsilon \lambda} \bar{v} & -t i
\end{array}\right) \\
& =\widehat{A d}_{a}\left(\begin{array}{cc}
0\left(e^{-2 \epsilon \lambda}-e^{2 \epsilon \lambda}\right) v \\
0
\end{array}\right) \\
& =\left(1-e^{4 \epsilon \lambda}\right)\left(\begin{array}{cc}
0 & v \\
0 & 0
\end{array}\right)
\end{aligned}
$$

so that

$$
\begin{aligned}
\frac{1}{\epsilon}\left\langle X, \widehat{A d}_{a} \rho_{\mathfrak{b}}\left(\widehat{A d}_{a^{-1}} Y\right)\right\rangle & =\frac{2}{\epsilon} \operatorname{Imtr}\left[\left(\begin{array}{cc}
s i & u \\
-\bar{u}-s i
\end{array}\right)\left(\begin{array}{cc}
0 & \left(1-e^{4 \epsilon \lambda}\right) v \\
0 & 0
\end{array}\right)\right] \\
& =-\frac{2}{\epsilon}\left(1-e^{4 \epsilon \lambda}\right) \operatorname{Im}(\bar{u} v) \\
& =\frac{2}{\epsilon}\left(e^{4 \epsilon \lambda}-1\right) \operatorname{Im}(\bar{u} v) .
\end{aligned}
$$

If we evaluate the right-hand side of the above formula we see

$$
\begin{aligned}
\frac{1}{2} \widehat{\pi}_{\Lambda}(a)\left(\widehat{d R}_{a^{-1}}^{*} \alpha_{X}, \widehat{d R}_{a^{-1}}^{*} \alpha_{Y}\right) & =\frac{1}{2 \epsilon}\left[\alpha_{X} \wedge \alpha_{Y}\left(\widehat{A d}_{a} E, \widehat{A d}_{a} i E\right)-\alpha_{X} \wedge \alpha_{Y}(E, i E)\right. \\
& =\frac{2}{\epsilon}\left[e^{4 \epsilon \lambda} \operatorname{Im}(\bar{u} v)-\operatorname{Im}(\bar{u} v)\right] \\
& =\frac{2}{\epsilon}\left(e^{4 \epsilon \lambda}-1\right) \operatorname{Im}(\bar{u} v) \\
& =\widehat{\pi}_{B_{\epsilon}}(a)\left(\widehat{d R}_{a^{-1}}^{*} \alpha_{X}, \widehat{d R}_{a^{-1}}^{*} \alpha_{Y}\right)
\end{aligned}
$$

We then have the following.

Corollary 5.25. $\widehat{\pi}_{B_{\epsilon}}(a)=\frac{1}{2 \epsilon}\left(1-e^{-4 \epsilon \lambda}\right) \widehat{d L}_{a}(E \wedge i E)$

Proof.

$$
\begin{aligned}
\widehat{\pi}_{B_{\epsilon}}(a) & =\frac{1}{2 \epsilon}\left[\widehat{d L}_{a}(E \wedge i E)-\widehat{d R}_{a}(E \wedge i E)\right] \\
& =\frac{1}{2 \epsilon} \widehat{d L}_{a}\left[E \wedge i E-\widehat{A d}_{a^{-1}}(E \wedge i E)\right] \\
& =\frac{1}{2 \epsilon} \widehat{d L}_{a}\left[E \wedge i E-e^{-4 \epsilon \lambda}(E \wedge i E)\right] \\
& =\frac{1}{2 \epsilon}\left(1-e^{-4 \epsilon \lambda}\right) \widehat{d L}_{a}(E \wedge i E)
\end{aligned}
$$


The diffeomorphism $\varphi_{\epsilon}: K / T \rightarrow \Sigma_{\lambda}^{\epsilon}$ gives us $\left(d \varphi_{\epsilon}\right)_{e}: \mathfrak{k} / \mathfrak{t} \rightarrow T_{a} \Sigma_{\lambda}^{\epsilon} \subset T_{a} B_{\epsilon}$ defined by $\left(d \varphi_{\epsilon}\right)_{e}(\xi)=\frac{1}{\epsilon} \widehat{d L}_{a} \rho_{b}\left(\widehat{A d}_{a^{-1}} \xi\right)$. Now let $X=\frac{1}{2}\left(\begin{array}{cc}0 & 1 \\ -1 & 0\end{array}\right)$ and $Y=\frac{1}{2}\left(\begin{array}{cc}0 & i \\ i & 0\end{array}\right)$ as in $\S 5.1$, then

$$
\left(d \varphi_{\epsilon}\right)_{e}(X)=\frac{1}{2 \epsilon}\left(e^{-2 \epsilon \lambda}-e^{2 \epsilon \lambda}\right) \widehat{d L}_{a} E \text { and }\left(d \varphi_{\epsilon}\right)_{e}(Y)=\frac{1}{2 \epsilon}\left(e^{-2 \epsilon \lambda}-e^{2 \epsilon \lambda}\right) \widehat{d L}_{a} i E .
$$

It then follows that

LeMmA 5.26. $\left(d \varphi_{\epsilon}\right)_{e}\left(\pi_{\lambda, \epsilon}(e)\right)=\widehat{\pi}_{B_{\epsilon}}(a)$

Proof.

$$
\begin{aligned}
\left(d \varphi_{\epsilon}\right)_{e}\left(\pi_{\lambda, \epsilon}(e)\right) & =\left(d \varphi_{\epsilon}\right)_{e}\left(\epsilon\left(\pi_{\infty}(e)-\tau(\epsilon \lambda) \pi_{0}(e)\right)\right) \\
& =\epsilon\left(d \varphi_{\epsilon}\right)_{e}\left(\pi_{\infty}(e)\right)-\epsilon \tau(\epsilon \lambda)\left(d \varphi_{\epsilon}\right)_{e}\left(\pi_{0}(e)\right) \\
& =0-2 \epsilon \tau(\epsilon \lambda)\left(d \varphi_{\epsilon}\right)_{e}(X \wedge Y) \\
& =-\frac{1}{2 \epsilon} \tau(\epsilon \lambda)\left(e^{-2 \epsilon \lambda}-e^{2 \epsilon \lambda}\right)^{2} \widehat{d L}_{a}(E \wedge i E) \\
& =-\frac{1}{2 \epsilon}\left(e^{-4 \epsilon \lambda}-1\right) \widehat{d L}_{a}(E \wedge i E) \\
& =\widehat{\pi}_{B_{\epsilon}}(a)
\end{aligned}
$$

This completes the proof of Proposition 5.23.

We can next look at the product $(K / T)^{n}$. We give $(K / T)^{n}$ the product Poisson structure $\pi_{\lambda, \epsilon}=\pi_{\lambda_{1}, \epsilon}+\cdots+\pi_{\lambda_{n}, \epsilon}$. Define the map

$$
\widetilde{\Phi}_{\epsilon}:(K / T)^{n} \rightarrow \Sigma_{\lambda_{1}}^{\epsilon} \times \cdots \times \Sigma_{\lambda_{n}}^{\epsilon}
$$

given by

$$
\widetilde{\Phi}_{\epsilon}\left(k_{1}, \ldots, k_{n}\right)=\left(\varphi_{1}^{\epsilon}\left(k_{1}\right), \ldots, \varphi_{n}^{\epsilon}\left(k_{n}\right)\right)=\left(\widehat{D}_{\epsilon}^{\ell}\left(k_{1}\right)\left(a_{\lambda_{1}}\right), \ldots, \widehat{D}_{\epsilon}^{\ell}\left(k_{n}\right)\left(a_{\lambda_{n}}\right)\right)
$$

where $\varphi_{i}^{\epsilon}\left(k_{i}\right)=\widehat{D}_{\epsilon}^{\ell}\left(k_{i}\right)\left(a_{\lambda_{i}}\right)$ and $a_{\lambda_{i}}=\exp ^{\epsilon}\left(\lambda_{i} H\right) \in B_{\epsilon}$. We note the map $\widetilde{\Phi}_{\epsilon}$ : $(K / T)^{n} \rightarrow \Sigma_{\lambda_{1}}^{\epsilon} \times \cdots \times \Sigma_{\lambda_{n}}^{\epsilon}$ is a symplectomorphism.

We leave the proof of the following lemma to the reader.

LEMMA 5.27. The action of $K$ on $(K / T)^{n}$ given by

$$
k \circ\left(k_{1}, \ldots, k_{n}\right)=\left(k k_{1}, \rho_{K}\left(k \varphi_{1}^{\epsilon}\left(k_{1}\right)\right) k_{2}, \ldots, \rho_{K}\left(k \varphi_{1}^{\epsilon}\left(k_{1}\right) \cdots \varphi_{n-1}^{\epsilon}\left(k_{n-1}\right)\right) k_{n}\right)
$$

is the pull back under $\widetilde{\Phi}_{\epsilon}$ of the $\epsilon$-dressing action on $\Sigma_{\lambda_{1}}^{\epsilon} \times \cdots \times \Sigma_{\lambda_{n}}^{\epsilon} \subset B_{\epsilon}^{n}$.

The momentum map for the action of $\left(K, \epsilon \pi_{K}\right)$ on $\left((K / T)^{n}, \pi_{\lambda, \epsilon}\right)$ is

$$
\widetilde{\Psi}_{\epsilon}:(K / T)^{n} \rightarrow B_{\epsilon}
$$

where

$$
\widetilde{\Psi}_{\epsilon}\left(k_{1}, . ., k_{n}\right)=\varphi_{1}^{\epsilon}\left(k_{1}\right) * \cdots * \varphi_{n-1}^{\epsilon}\left(k_{n-1}\right) .
$$


5.3. The $\epsilon$-dressing orbits are small spheres in hyperbolic 3-space. Let $b$ be the Killing form on $\mathfrak{g}$ divided by 8 . We have normalized $b$ so that the induced Riemannian metric (, ) on $G / K$ has constant curvature -1 . We let $b_{\epsilon}=f_{\epsilon}^{*} b$, hence $b_{\epsilon}$ is the Killing form on $\mathfrak{g}_{\epsilon}$. Then $(,)_{\epsilon}=F_{\epsilon}^{*}($,$) is the induced Riemannian metric on$ $G_{\epsilon} / K$ and $G_{\epsilon} / K$ has constant curvature -1 (since $F_{\epsilon}$ is an isometry). We will call $(,)_{\epsilon}$ the hyperbolic metric on $G_{\epsilon} / K$.

The $\operatorname{map} \zeta: B_{\epsilon} \rightarrow G_{\epsilon} / K$ given by $\zeta(b)=b * K$ is a diffeomorphism that intertwines the $\epsilon$-dressing orbits of $K$ on $B_{\epsilon}$ with the natural $K$ action on $G_{\epsilon} / K$ given by left multiplication (using the multiplication in $G_{\epsilon}$ ). We abbreviate the identity coset $K$ in $G_{\epsilon} / K$ to $x_{0}$ and use the same letter for the corresponding point in $G / K$. We have

LEMMA 5.28. The image of the $\epsilon$-dressing orbit $\Sigma_{\lambda}^{\epsilon}$ under $\zeta$ is the sphere around $x_{0}$ of radius $\epsilon \lambda$.

Proof. Let $d_{\epsilon}$ be the Riemannian distance function on $G_{\epsilon} / K$ and $d$ the Riemannian distance function on $G / K$. We have

$$
\begin{aligned}
d_{\epsilon}\left(x_{0}, \exp _{x_{0}}^{\epsilon} \lambda H\right) & =d\left(x_{0}, F_{\epsilon} \exp _{x_{0}}^{\epsilon} \lambda H\right) \\
& =d\left(x_{0}, \exp _{x_{0}} f_{\epsilon}(\lambda H)\right) \\
& =d\left(x_{0}, \exp _{x_{0}} \epsilon \lambda H\right) \\
& =\epsilon \lambda
\end{aligned}
$$

5.4. The family of symplectic quotients. In this section we will continue to use the notation of $\S 5.2$. Let $p: E=(K / T)^{n} \times I \rightarrow I$ be a projection. Here we define $I=[0,1]$. We let $T^{v e r t}(E) \subset T(E)$ be the tangent space to the fibers of $p$. Hence $\bigwedge^{2} T^{v e r t}(E)$ is a subbundle of $\Lambda^{2} T(E)$. We define a Poisson bivector $\pi$ on $E$ by $\pi(u, \epsilon)=\left.\pi_{\lambda, \epsilon}\right|_{u} . \pi$ is a section of $\bigwedge^{2} T^{\text {vert }}(E)$.

Let $S \subset B^{n} \times I$ be defined by $S=\left\{(b, \epsilon) \mid b \in \Sigma_{\lambda}^{\epsilon}\right\}$. We let $\left(K, \epsilon \pi_{K}\right)$ act on $(K / T)^{n} \times I$ by $k \cdot(u, \epsilon)=(k \circ u, \epsilon)$, where $\circ$ is the action given in Lemma 5.27, and act on $S$ by $k \cdot(b, \epsilon)=\left(\widehat{D}_{\epsilon}^{\ell}(k)(b), \epsilon\right)$. We then define the map $\Phi: E \rightarrow S$ by $\Phi(u, \epsilon)=\left(\widetilde{\Phi}_{\epsilon}(u), \epsilon\right)$ which is a K-equivariant diffeomorphism. We also define $\Psi: E \rightarrow B$ by $\Psi(u, \epsilon)=\widetilde{\Psi}_{\epsilon}(u)$.

REMARK 5.29. $\left.\Psi\right|_{p^{-1}(\epsilon)}$ is the momentum map for the Poisson action of $\left(K, \epsilon \pi_{K}\right)$ on $(K / T)^{n} \times\{\epsilon\}$.

We need some notation. Suppose $n \geq m, F: \mathbb{R}^{n+1} \rightarrow \mathbb{R}^{m}$ is a smooth map, and $0 \in \mathbb{R}^{m}$ is a regular value of $F$. Let $M=F^{-1}(0)$. Write $\mathbb{R}^{n+1}=\mathbb{R}^{n} \times \mathbb{R}$ with $x \in \mathbb{R}^{n}, t \in \mathbb{R}$. Let $p: \mathbb{R}^{n+1} \rightarrow \mathbb{R}$ be the projection onto the $t$-line. The next lemma is taken from [Sa].

LEMMA 5.30. Let $(x, t) \in M$. Suppose $\left.\frac{\partial F}{\partial x}\right|_{(x, t)}$ has maximal rank $m$. Then $\left.d p\right|_{(x, t)}: T_{(x, t)}(M) \rightarrow T_{t}(\mathbb{R})$ is onto.

Proof. It suffices to construct a tangent vector $v \in T_{(x, t)}\left(\mathbb{R}^{n+1}\right)$ satisfying

(i) $\left.v \in \operatorname{ker} d F\right|_{(x, t)}$

(ii) $v=\sum_{i=1}^{n} c_{i} \frac{\partial}{\partial x_{i}}+\frac{\partial}{\partial t}$ 
Put $c=\left(c_{1}, \ldots, c_{n}\right)$ and write the Jacobian matrix $\left.d F\right|_{(x, t)}$ as $(A, b)$ where $A$ is the $m$ by $n$ matrix given by $A=\left.\frac{\partial F}{\partial x}\right|_{(x, t)}$ and $b$ is the column vector of length $m$ given by $b=\left.\frac{\partial F}{\partial t}\right|_{(x, t)}$. We are done if we can solve

$$
A c+b=0 \text {. }
$$

But since $A: \mathbb{R}^{n} \rightarrow \mathbb{R}^{m}$ is onto we can solve this equation.

REMARK 5.31. We need to generalize to the case in which $\mathbb{R}^{n+1}$ is replaced by the closed half-space $\bar{H}=\left\{(x, t): x \in \mathbb{R}^{n}, t \geq 0\right\}$ and the $t$-line by the closed half-line. Given $(x, 0) \in \partial M$ we wish to find $v=\sum_{i=1}^{n} c_{i} \frac{\partial}{\partial x_{i}}+\frac{\partial}{\partial t}$ with $\left.d F\right|_{(x, o)}(v)=0$ (so $v$ is in the tangent half-space to $M$ at $(x, 0) \in \partial M)$. The argument is analogous to that of the lemma and is left to the reader.

CoRollary 5.32. Suppose $M$ is compact and for all $(x, t)$ and further that $\left.\frac{\partial F}{\partial x}\right|_{(x, t)}$ has maximal rank for all $(x, t) \in M$. Then $p: M \rightarrow \mathbb{R}$ is a trivial fiber bundle.

Proof. $p$ is proper since $M$ is compact. This is the Ehresmann fibration theorem [BJ, 8.12]. $\square$

REMARK 5.33. We leave to the reader the task of extending the corollary to the case where the $t$-line is replaced by the closed $t$ half-line.

We now return to our map $\Psi:(K / T)^{n} \times I \rightarrow B$. We have

$$
\Psi(u, \epsilon)=\varphi_{1}^{\epsilon}\left(u_{1}\right) * \cdots * \varphi_{n}^{\epsilon}\left(u_{1}\right) .
$$

Let $\epsilon>0$. We apply Lemma 2.5 to deduce that $1 \in B$ is a regular value for $u \rightarrow \Psi(u, \epsilon)$ (recall we have assumed $r$ is not on a wall of $D_{n}$ ). Now let $\epsilon=0$. It is immediate (see [KM2]) that $0 \in \mathbb{R}^{3}$ is a regular value of $u \rightarrow \Psi(u, \epsilon)$ (again because $r$ is not on a wall of $D_{n}$ ). We obtain

LEMMA 5.34. $p: \Psi^{-1}(1) \rightarrow I$ is a trivial fiber bundle.

Now let $\mathcal{M}=\Psi^{-1}(1) / K$. We note that $p$ factors through the free action of $K$ on $\Psi^{-1}(1)$ and we obtain a fiber bundle $\bar{p}: \mathcal{M} \rightarrow I$. This gives the required family of symplectic quotients.

Proposition 5.35. $\bar{p}: \mathcal{M} \rightarrow I$ is a trivial fiber bundle.

REMARK 5.36. $\bar{p}^{-1}(0)=M_{r}\left(\mathbb{E}^{3}\right)$ and $\bar{p}^{-1}(1)=M_{r}\left(\mathbb{H}^{3}\right)$ and we may identify $\mathcal{M}$ with the product $M_{r}\left(\mathbb{E}^{3}\right) \times I$.

We now give a description of the symplectic form along the fibers of $\bar{p}$. Recall that if $\pi: E \rightarrow B$ is a smooth fiber bundle then the relative forms on $E$ are the elements of the quotient of $A^{\bullet}(E)$ by the ideal generated by elements of positive degree in $\pi^{*} A^{\bullet}(B)$. Note the restriction of a relative form to a fiber of $\pi$ is well-defined and the relative forms are a differential graded-commutative algebra with product and differential induced by those of $A^{\bullet}(E)$.

Lu's one parameter family of forms $\omega_{\epsilon}$ of $\S 5.1$ induces a relative 2-form $\omega_{\epsilon}$ on $K / T \times I$ which is relatively closed. By taking sums we obtained a relative 2-form $\widetilde{\omega}_{\epsilon}$ on $(K / T)^{n} \times I$ and by restriction and projection a relative 2-form $\bar{\omega}_{\epsilon}$ on $\mathcal{M}$. Clearly $\bar{\omega}_{\epsilon}$ is relatively closed and induces the symplectic form along the fibers of $\bar{p}: \mathcal{M} \rightarrow I$.

We let $\left[\bar{\omega}_{\epsilon}\right]$ be the class in $H^{2}\left(p^{-1}(\epsilon)\right)$ determined by $\bar{\omega}_{\epsilon}$. 
5.5. $\left[\bar{\omega}_{\epsilon}\right]$ is constant and Moser's Theorem. To complete our proof we need to review the $i$-th cohomology bundle associated to a smooth fiber bundle $\pi: E \rightarrow B$. The total space $\mathcal{H}_{E}^{i}$ of the $i$-th cohomology bundle is given by $\mathcal{H}_{E}^{i}=\{(b, z): b \in$ $\left.B, z \in H^{i}\left(p^{-1}(b)\right)\right\}$. We note that a trivialization of $\left.E\right|_{U}$ induces an isomorphism between $\mathcal{H}_{\left.E\right|_{U}}^{i}$ and $\mathcal{H}_{U \times F}^{i}$. But $\mathcal{H}_{U \times F}^{i}=\left\{(x, z): x \in U, z \in H^{i}(F)\right\}$, whence $\mathcal{H}_{U \times F}^{i}=U \times H^{i}(F)$. It is then clear that $\mathcal{H}_{E}^{i}$ is a vector bundle over $B$ with typical fiber $H^{i}(F)$. We next observe that the action of the transition functions of $E$ on $H^{i}(F)$ induce the transition functions of $\mathcal{H}_{E}^{i}$. Hence if we trivialize $E$ relative to a covering $\mathcal{U}=\left\{U_{i}: i \in I\right\}$ such that all pairwise intersections are contractible then the corresponding transition functions of $\mathcal{H}_{E}^{i}$ are constant. Hence $\mathcal{H}_{E}^{i}$ admits a flat connection called the Gauss-Manin connection. We observe that a cross-section of $\mathcal{H}_{E}^{i}$ is parallel for the Gauss-Manin connection if when expressed locally as an element of $\mathcal{H}_{U \times F}^{i}$ as above it corresponds to a constant map from $U$ to $H^{i}(F)$.

REMARK 5.37. If $\tau$ is a relative $i$-form on $E$ which is relatively closed then it gives rise to a cross-section $[\tau]$ of $\mathcal{H}_{E}^{i}$ such that $[\tau](b)$ is the de Rham cohomology class of $\left.\tau(b)\right|_{\pi^{-1}(b)}$.

We now consider the relative 2 -form $\bar{\omega}_{\epsilon}$ on $\mathcal{M}$. The form $\bar{\omega}_{\epsilon}$ is obtained from the corresponding form $\widetilde{\omega}_{\epsilon}$ on $(K / T)^{n} \times I$ by first pulling $\widetilde{\omega}_{\epsilon}$ back to $\Psi^{-1}(1)$ then using the invariance of $\widetilde{\omega}_{\epsilon}$ under $K$ to descend $\widetilde{\omega}_{\epsilon}$ to $\bar{\omega}_{\epsilon}$. We observe that $\left[\widetilde{\omega}_{\epsilon}\right]$ (reps. $\left[\bar{\omega}_{\epsilon}\right]$ ) is a smooth section of $\mathcal{H}_{(K / T)^{n} \times I}^{2}\left(\right.$ resp. $\left.\left.\mathcal{H}_{\mathcal{M}}^{2}\right)\right)$.

We obtain a diagram of second cohomology bundles with connection

$$
\mathcal{H}_{(K / T)^{n} \times I}^{2} \stackrel{i^{*}}{\longrightarrow} \mathcal{H}_{\Psi^{-1}(1)}^{2} \stackrel{\pi^{*}}{\longleftarrow} \mathcal{H}_{\mathcal{M}}^{2}
$$

where $i: \Psi^{-1}(1) \rightarrow(K / T)^{n} \times I$ is the inclusion and $\pi: \Psi^{-1}(1) \rightarrow \mathcal{M}$ is the quotient map. We have

$$
\pi^{*}\left[\bar{\omega}_{\epsilon}\right]=i^{*}\left[\widetilde{\omega}_{\epsilon}\right]
$$

Proposition 5.38. $\left[\bar{\omega}_{\epsilon}\right]$ is parallel for the Gauss-Manin connection on $\mathcal{H}_{\mathcal{M}}^{2}$.

Proof. By Lemma 5.7, $\left[\widetilde{\omega}_{\epsilon}\right]$ is parallel for the Gauss-Manin connection on $(K / T)^{n} \times I$. Hence $i^{*}\left[\widetilde{\omega}_{\epsilon}\right]$ is parallel for the Gauss-Manin connection on $\mathcal{H}_{\Psi^{-1}(1)}^{2}$. But an elementary spectral sequence argument for the bundle $K \rightarrow \Psi^{-1}(1) \rightarrow \mathcal{M}$ shows that $\pi^{*}: \mathcal{H}_{\mathcal{M}}^{2} \rightarrow \mathcal{H}_{\Psi^{-1}(1)}^{2}$ is a bundle monomorphism. Hence if $\pi^{*}\left[\bar{\omega}_{\epsilon}\right]$ is parallel, so is $\left[\bar{\omega}_{\epsilon}\right]$.

CoRollary 5.39. The cohomology class of $\bar{\omega}_{\epsilon}$ is constant relative to any trivialization of $p: \mathcal{M} \rightarrow I$.

We now complete the proof of symplectomorphism by applying a version of Moser's Theorem [Mo] with $M=M_{r}\left(\mathbb{\Xi}^{3}\right)$. For the benefit of the reader we will state and prove the version of Moser's Theorem we need here.

THEOREM 5.40. Suppose $\omega_{\epsilon}$ is a smooth one-parameter family of symplectic forms on a compact smooth manifold $M$. Suppose the cohomology class $\left[\omega_{\epsilon}\right]$ of $\omega_{\epsilon}$ in $H^{2}(M)$ is constant. Then there is a smooth curve $\phi_{\epsilon}$ in Diff(M) with $\phi_{0}=i d_{M}$ such that

$$
\omega_{\epsilon}=\phi_{\epsilon}^{*} \omega_{0}
$$


Proof. Choose a smooth one-parameter family of 1-forms $\tau_{\epsilon}$ such that

$$
\frac{d \omega_{\epsilon}}{d \epsilon}=-d \tau_{\epsilon} .
$$

(We may choose $\tau_{\epsilon}$ smoothly by first choosing a Riemannian metric then taking $\tau_{\epsilon}$ to be the coexact primitive of $\frac{d \omega_{\epsilon}}{d \epsilon}$ - here we use the compactness of $M$ ).

Let $\zeta_{\epsilon}$ be the one parameter family of vector fields such that

$$
i_{\zeta_{\epsilon}} \omega_{\epsilon}=\tau_{\epsilon} .
$$

Now we integrate the time dependent vector field $\zeta_{\epsilon}$ to a family $\Psi_{\epsilon}$ of diffeomorphisms (again we use that $M$ is compact). We have

$$
\begin{aligned}
\frac{d}{d \epsilon} \Psi_{\epsilon}^{*} \omega_{\epsilon} & =\Psi_{\epsilon}^{*} \mathcal{L}_{\zeta_{\epsilon}} \omega_{\epsilon}+\Psi_{\epsilon}^{*} \frac{d \omega_{\epsilon}}{d \epsilon} \\
& =\Psi_{\epsilon}^{*}\left[d \iota_{\zeta_{\epsilon}} \omega_{\epsilon}-d \tau_{\epsilon}\right] \\
& =\Psi_{\epsilon}^{*}\left[d \tau_{\epsilon}-d \tau_{\epsilon}\right] \\
& =0 .
\end{aligned}
$$

Hence $\Psi_{\epsilon}^{*} \omega_{\epsilon}$ is constant so $\Psi_{\epsilon}^{*} \omega_{\epsilon}=\omega_{0}$ and $\omega_{\epsilon}=\left(\Psi_{\epsilon}^{-1}\right)^{*} \omega_{0}$.

5.6. The geometric meaning of the family $\mathcal{M}$ of symplectic quotients shrinking the curvature. We recall that $X_{\kappa}$ denotes the complete simply-connected Riemannian manifold of constant curvature $\kappa$. Let $r=\left(r_{1}, r_{2}, \ldots, r_{n}\right) \in\left(\mathbb{R}_{+}\right)^{n}$ with $r$ not on a wall of $D_{n}$. Let $M_{r}\left(X_{\kappa}\right)$ be the moduli space of n-gon linkages with side-lengths $r$ in the space $X_{\kappa}$. The following theorem is the main result of [Sa].

THEOREM 5.41. There exists $\alpha>0$ and an analytically trivial fiber bundle $\pi: \mathcal{E} \rightarrow(-\infty, \alpha)$ such that $\pi^{-1}(\kappa)=M_{r}\left(X_{\kappa}\right)$.

Let $\mathcal{M}$ be the family of symplectic quotients just constructed (except we will take $(-\infty, 0]$ as base instead of $[1,0])$. We then have

THEOREM 5.42. We have an isomorphism of fiber bundles

$$
\left.\mathcal{E}\right|_{(-\infty, 0]} \simeq \mathcal{M} .
$$

We will need

LEMMA 5.43. Let $\lambda>0$. Then we have a canonical isomorphism

$$
M_{r}\left(X_{\kappa}\right) \simeq M_{\lambda r}\left(X_{\kappa / \lambda}\right) .
$$

Proof. Multiply the Riemannian metric on $X_{\kappa}$ by $\lambda$. Then the Riemannian distance function is multiplied by $\lambda$ and the sectional curvature is multiplied by $\frac{1}{\lambda}$.

REMARK 5.44. There is a good way to visualize the above isomorphism by using the embedding of $X_{\kappa}, \kappa<0$, in Minkowski space (as the upper sheet of the hyperboloid 
$\left.x^{2}+y^{2}+z^{2}-t^{2}=\frac{-1}{\kappa^{2}}\right)$ or $X_{\kappa}, \kappa>0$ in $\mathbb{E}^{4}$ (as the sphere $x^{2}+y^{2}+z^{2}+t^{2}=\frac{1}{\kappa^{2}}$ ). The dilation map $v \mapsto \lambda v$ of the ambient vector space maps $X_{\kappa}$ to $X_{\kappa / \lambda}$ and multiplies the side-lengths by $\lambda$.

Now we can prove the theorem. Let $\bar{p}: \mathcal{M} \rightarrow(-\infty, 0]$ be the family constructed is $\S 5.4$. By Lemma 5.28 we see that $\bar{p}^{-1}(\epsilon) \simeq M_{\epsilon r}\left(X_{-1}\right)$. Thus we are shrinking the side-lengths of the n-gons as $\epsilon \rightarrow 0$. But we have just constructed a canonical isomorphism

$$
M_{\epsilon r}\left(X_{-1}\right) \simeq M_{r}\left(X_{-\epsilon}\right) .
$$

So we may regard the deformation of $\S 5.4$ as keeping the side-lengths fixed and shrinking the curvature to zero.

To give a formal proof we will construct an explicit diffeomorphism

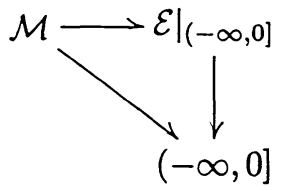

To this end, observe that the map $B \times(-\infty, 0] \rightarrow G / K \times(-\infty, 0]$ given by $(b, \kappa) \mapsto(b * K, \kappa)$ induces a $K$-equivariant diffeomorphism

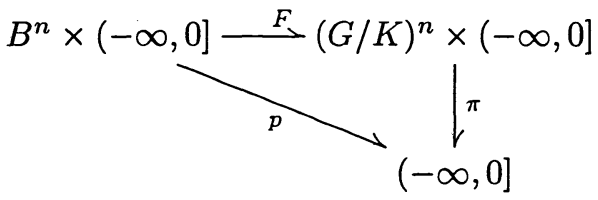

given by $F\left(\left(b_{1}, \ldots, b_{n}\right), \kappa\right)=\left(\left(K, b_{1} * K, \ldots, b_{1} * \cdots * b_{n-1} * K\right), \kappa\right)$.

We give $\pi^{-1}(\kappa)$ the Riemannian metric $|\kappa|(,)_{\kappa}$. Let $\widetilde{\mathcal{E}}^{\prime} \subset(G / K)^{n} \times(-\infty, 0]$ be defined by

$$
\widetilde{\mathcal{E}}^{\prime}=\left\{\left(y_{1}, \ldots, y_{n}, \kappa\right): y_{1}=x_{0}, d_{\kappa}\left(y_{i}, y_{i+1}\right)=r_{i}, 1 \leq i \leq n\right\}
$$

Here $d_{\kappa}$ is the distance function on $\pi^{-1}(\kappa)$ associated to the Riemannian metric $|\kappa|(,)_{\kappa}$. Let $\Sigma_{r} \times(-\infty, 0]$ be the dressing orbit through $\left(e^{r_{1} H}, \ldots, e^{r_{n} H}\right)$ for the $K$ dressing action of $K$ on $p^{-1}(\kappa)$. We let $\widetilde{M} \subset \Sigma_{r} \times(-\infty, 0]$ be the subset $\widetilde{M}=$ $\left\{\left(b_{1}, \ldots, b_{n}, \kappa\right): b_{1} * b_{2} * \cdots * b_{n}=1\right\}$. Then $F$ carries $\widetilde{\mathcal{M}}$ diffeomorphically onto $\widetilde{\mathcal{E}}^{\prime}$ and induces the required diffeomorphism $\left.\mathcal{M} \rightarrow \mathcal{E}\right|_{(-\infty, 0]}$.

REMARK 5.45. The relative 2-form $\bar{\omega}_{\epsilon}$ is a symplectic form along the fibers of $\bar{p}$. Thus we have made the restriction of the family of [Sa] to $(-\infty, 0]$ into a family of symplectic manifolds. Can $\omega_{\epsilon}$ be extended to $(-\infty, \alpha)$ for some $\alpha>0$ ?

\section{REFERENCES}

[A] A. Alekseev, On Poisson actions of compact Lie groups on symplectic manifolds, J. Differential Geom., 45 (1997), pp. 241-256. 
[BGS] W. Ballmann, M. Gromov, ANd V. Schroeder, Manifolds of Nonpositive Curvature, Progress in Math. 61, Birkhauser, 1985.

[BJ] T. BRÖCKER AND K. JÄNICH, Introduction to Differential Topology, Cambridge University Press, 1982.

[CP] V. Chari and A. Pressley, A Guide to Quantum Groups, Cambridge Univ. Press, 1994.

[DE] A. DOUADY AND C. EARLE, Conformally natural extension of homeomorphisms of the circle, Acta Math., 157 (1986), pp. 23-48.

[DF] J. Dollard AND C. Friedman, Product Integration, Encyclopedia of Mathematics and its Applications 10, Addison-Wesley, 1979.

[DM] P. DeLIGNE AND G. Mostow, Monodromy of hypergeometric functions and non-lattice integral monodromy, Publications of IHES, 63 (1986), pp. 5-90.

[FM] H. FlaschKa AND J.J. Millson, On the moduli space of $n$ points in $\mathbb{C P P}^{m}$, in preparation.

[FR] H. FlaschKa AND T. RATIU, A convexity theorem for Poisson actions of compact Lie groups, Ann. Sci. Ecole Norm. Sup. (4), 29:6 (1996), pp. 787-809.

[Gu] V. Gulllemin, Moment Maps and Combinatorial Invariants of Hamiltonian $T^{n}$-spaces, Progess in Math. 122, Birkhäuser, 1994.

[GW] V. Ginzburg AND A. WeInStein, Lie-Poisson structure on some Poisson Lie groups, J. AMS, 5 (1992), pp. 445-453.

[HK] J.-C. HaUsmann AND A. KnUtson, Polygon spaces and Grassmannians, Enseign. Math. (2), 43:1-2 (1997), pp. 173-198.

[J] L. JEFFREY, Extended moduli spaces of flat connections on Riemann surfaces, Math. Ann., 298 (1994), pp. 667-692.

[KK] E. KLASSEN AND P. KIRK, Representation spaces of Seifert fibered homology spheres, TopolOgy, 30 (1990), pp. 77-95.

[KM1] M. KAPOVICH AND J.J. Millson, On the moduli space of polygons is the Euclidean plane, J. Differential Geom., 42 (1995), pp. 133-164.

[KM2] M. KAPOVICH AND J.J. MILLSON, The symplectic geometry of polygons in Euclidean space, J. Differential Geom., 44 (1996), pp. 479-513.

[KN] G. KEMPF AND L. NESS, The length of vectors in representation spaces, Algebraic Geometry, Proceedings, Copenhagen 1978, Lecture Notes in Math. 732, Springer, 1978, pp. 233244.

[Ki] F. KIRWAN, Cohomology of Quotients in Symplectic and Algebraic Geometry, Mathematical Notes, Princeton University Press, 1984.

[Lu1] J.-H. LU, Multiplicative and affine Poisson structures on Lie groups, Ph.D. thesis, University of California, Berkeley, 1990.

[Lu2] J.-H. LU, Classical dynamical $r$-matrices and homogeneous Poisson structures on $G / H$ and $K / T$, preprint math.SG9909004.

[Lu3] J.-H. LU, Momentum mappings and reduction of Poisson actions, in Symplectic Geometry, Groupoids, and Integable Systems, MSRI Publ. 20, Springer-Verlag, New York, 1991, pp. 209-226.

[LR] J.-H. LU AND T. RATIU, On the nonlinear convexity theorem of Kostant, Journal of AMS, 4:2 (1991), pp. 349-363.

[LW] J.-H. LU AND A. WeINSTEIN, Poisson Lie groups, dressing transformations, and Bruhat decompositions, J. Differential Geom., 31:2 (1990), pp. 501-526.

[Mo] J. MOSER, On the volume elements on a manifold, Trans. Amer. Math. Soc., 120 (1965), pp. 286-294.

[MZ] J. J. Millson AND B. ZoMBro, A Kähler structure on the moduli space of isometric maps of a circle into Euclidean space, Invent. Math., 123 (1996), pp. 35-59.

[Sa] M. SARGENT, Diffeomorphism equivalence of configuration spaces of polygons in constant curvature spaces, Ph.D. thesis, University of Maryland, 1995.

[STS] M. Semenov-Tian-Shansky, Dressing transformations and Poisson group actions, Publ. of RIMS, 21 (1985), pp. 1237-1260.

[Tr] T. TRELOAR, The symplectic geometry of polygons in the 3-sphere, preprint, 2000.

[Wa] F. WARNer, Foundations of Differentiable Manifolds and Lie Groups, Scott, Foresman and Company, 1971. 